UCRL-ID-123486

\title{
Hydrologic Resources Management Program FY 1995 Progress Report
}

\author{
David K. Smith \\ Bradley K. Esser \\ Jacqueline M. Kenneally \\ Richard J. Nagle \\ jo Ainn ñ. T̃ego \\ Jennifer L. Royval
}

RECEIVED MAY 151996

OSTI

\section{March 1996}
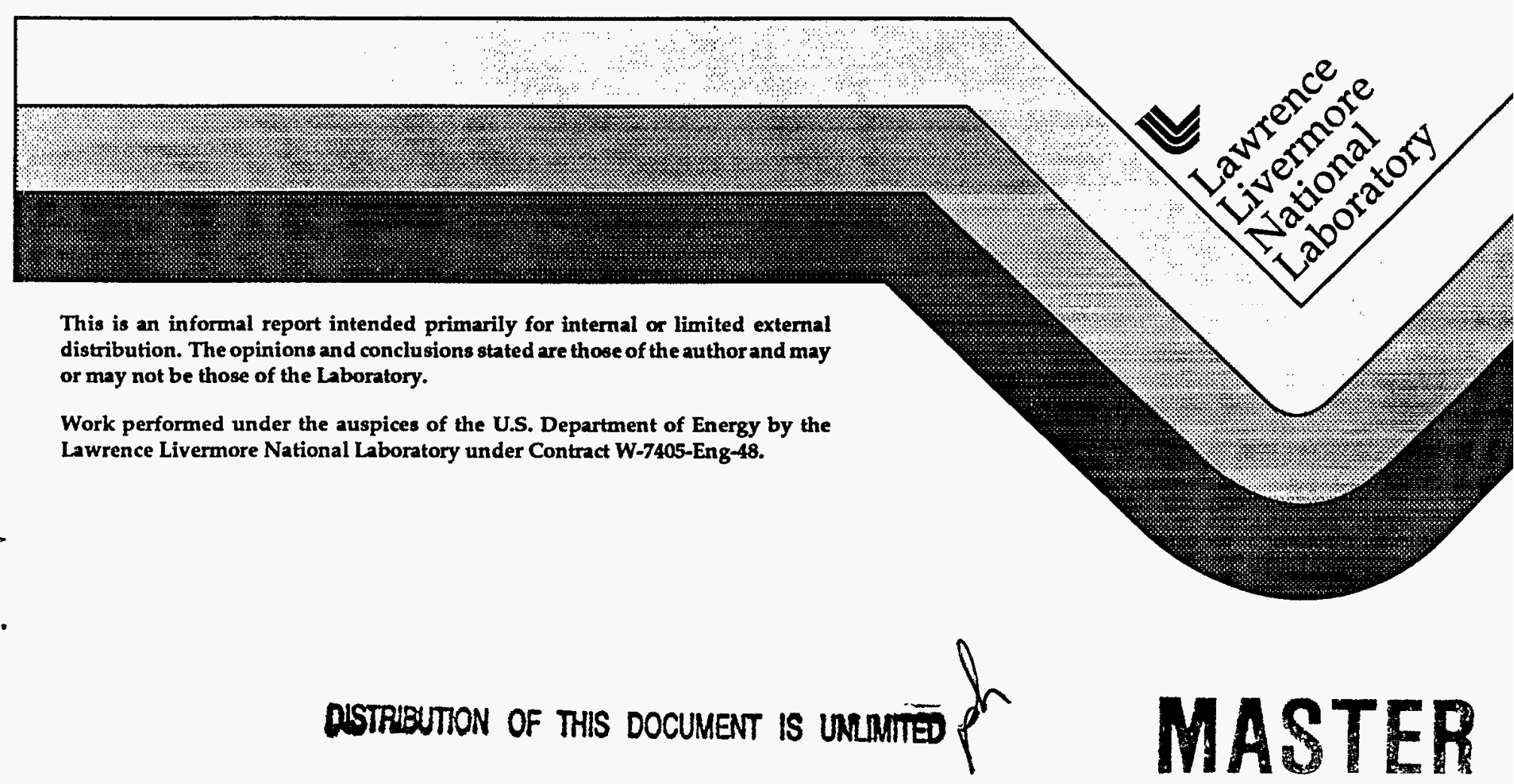


\section{DISCLAIMER}

This document was prepared as an account of work sponsored by an agency of the United States Government. Neither the United States Government nor the University of California nor any of their employees, makes any warranty, express or implied, or assumes any legal liability or responsibility for the accuracy, completeness, or usefulness of any information, apparatus, product, or process disclosed, or represents that its use would not infringe privately owned rights. Reference herein to any specific commercial products, process, or service by trade name, trademark, manufacturer, or otherwise, does not necessarily constitute or imply its endorsement, recommendation, or favoring by the United States Government or the University of California. The views and opinions of authors expressed herein do not necessarily state or reflect those of the United States Government or the University of California, and shall not be used for advertising or product endorsement purposes.

This report has been reproduced directly from the best available copy.

Available to DOE and DOE contractors from the Office of Scientific and Technical Information P.O. Box 62, Oak Ridge, TN 37831

Prices available from (615) 576-8401, FTS 626-8401

Available to the public from the

National Technical Information Service

US. Department of Commerce

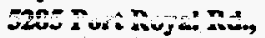

Springfield, VA 22161 


\section{DISCLAMIER}

Portions of this document may be illegible in electronic image products. Images are produced from the best available original document. 
HYDROLOGIC RESOURCES MANAGEMENT PROGRAM FY 1995 PROGRESS REPORT

Compiled by David K. Smith

Contributors:

Bradley K. Esser

Jacqueline M. Kenneally

Richard J. Nagle

Jo Ann H. Rego

Isotope Sciences Division

Lawrence Livermore National laboratory

Jennifer L. Royval

Stanford University

March, 1996 


\section{EXECUTIVE SUMMARY}

This report presents the results of FY 1995 technical studies conducted by the Lawrence Livermore National Laboratory (LLNL) as part of the Hydrology and Radionuclide Migration Program (HRMP), a multi-agency program sponsored by the U.S. Department of Energy, Nevada Operations Office (DOE/NV), to address the environmental consequences of nuclear weapons testing at the Nevada Test Site (NTS). A priority is to better characterize the complex near-field environment in order to assess and predict the movement of radionuclides in groundwater. Other participating organizations include the Los Alamos National Laboratory (LANL), the U.S. Geological Survey (USGS) and the Desert Research Institute (DRI) of the University of Nevada.

A radiologic source term in excess of $10^{8}$ curies of tritium, fission products, activation products and actinides is residual from more than three decades of underground nuclear weapons testing at the Nevada Test Site (NTS). Burial depths to insure containment of these explosions necessitated firing approximately one third of the more than 800 underground nuclear tests within one cavity radius or below the static water table. Work at LLNL has focused on studies of radionuclide transport under saturated, partially saturated or unsaturated conditions as well as investigations of the stable, radiogenic and cosmogenic isotope systematics of NTS groundwaters. LLNL has prioritized these studies because of the significance for potential radionuclide migration at the Nevada Test Site. LLNL utilizes expertise in nuclear weapons testing, radiochemical diagnostics, nuclear test phenomenology, mass spectrometry, aqueous geochemistry and field and laboratory studies of radionuclide migration to bring a unique measurement and interpretative capability to this research.

The HRMP is organized into one administrative and three technical elements: program coordination, groundwater monitoring, operational support, and groundwater protection respectively. The LLNL-HRMP is arranged similarly. Results from our FY95 technical program are described in the following sections. The LLNL-HRMP groundwater monitoring task included a field program to sample surface and groundwaters along a regional flow path including known recharge and discharge centers off the NTS. The operational support task provided a compilation of radionuclide, inorganic and organic analyses of fluids returned from saturated nuclear test cavities. Groundwater protection activities emphasized the distribution and setting of radionuclides residual from underground nuclear weapon tests. 


\title{
I. GROUNDWATER MONITORING
}

\section{FY95 OFF-NEVADA TEST SITE GROUNDWATER SAMPLING}

\author{
Jacqueline M. Kenneally and Jo Ann H. Rego
}

\section{INTRODUCTION}

Concern over the potential for radionuclide migration under saturated conditions emphasizes an accurate measure of NTS groundwater flow paths and rates. Because dissolved natural isotope signatures can provide information about recharge conditions, ages of groundwater and groundwater flow paths, isotope investigations are an important component of studies of the regional groundwater flow system in southern Nevada.

During FY92-FY93, LLNL sampled and analyzed groundwater from over 30 wells at NTS as part of an isotope hydrology investigation of groundwater sources, flow rates and flow paths (Davisson et al., 1994). The analytes included environmental isotopes $\left({ }^{2} \mathrm{H} /{ }^{1} \mathrm{H},{ }^{18} \mathrm{O} /{ }^{16} \mathrm{O},{ }^{13} \mathrm{C},{ }^{14} \mathrm{C},{ }^{36} \mathrm{Cl}\right.$, noble gases) and test-derived radionuclides $\left({ }^{3} \mathrm{H},{ }^{90} \mathrm{Sr},{ }^{125} \mathrm{Sb},{ }^{60} \mathrm{Co},{ }^{99} \mathrm{Tc},{ }^{137} \mathrm{Cs}\right)$. Davisson et al. (1994) and Kenneally et al. (1995) concluded that groundwater at the NTS is recharged from the north and northeast. This is consistent with interpretations from earlier studies that relied on measurements of hydrologic gradient (e.g., Winograd and Thordarson, 1975).

Our FY92-FY93 results helped target off-site recharge and discharge centers for our FY95 sampling campaign. These areas include: Pahranagat Valley, Emigrant Valley, Ash Meadows, Oasis Valley and U.S. Air Force (USAF) lands north of the NTS (Figure 1). Groundwater from the Pahranagat Valley, north and east of the NTS, is thought to recharge the regional carbonate aquifer underlying the NTS (Winograd and Thordarson, 1975).

Groundwaters from the Ash Meadows, south and west of the NTS, represent discharge from the same regional flow system. Valleys north of the NTS (Kawich Valley, Gold Flat, Reveille Valley, Cactus Flat), under jurisdiction of the U.S. Air Force, were also targeted for groundwater sampling; however security, safety and logistical concerns precluded access in FY95.

In FY95 , LLNL collected samples from 19 springs and well sites outside the boundaries of the NTS (Figure 1 and Table I). Samples will be analyzed for ${ }^{2} \mathrm{H} /{ }^{1} \mathrm{H},{ }^{3} \mathrm{H},{ }^{18} \mathrm{O} /{ }^{16} \mathrm{O},{ }^{13} \mathrm{C},{ }^{14} \mathrm{C},{ }^{36} \mathrm{Cl},{ }^{129} \mathrm{I}$, noble gases, major cations and anions in FY96. Because sampling locations were far removed from historical nuclear test centers at the Nevada Test Site, samples were not collected for analysis of radionuclides. 


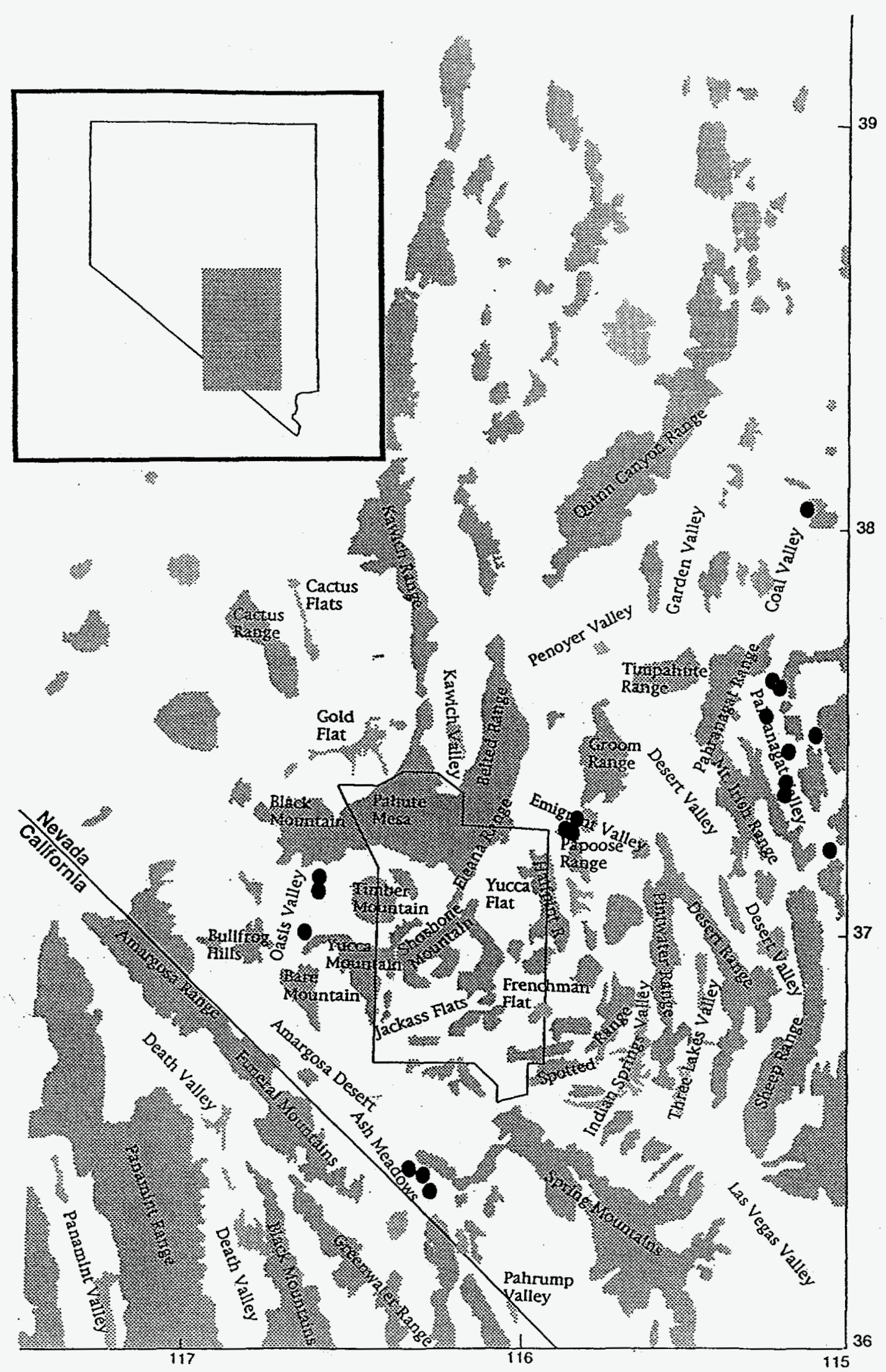

Figure 1: FY94 and FY95 sampling sites outside the boundaries of the NTS. 
TABLE I

\begin{tabular}{|c|c|c|c|c|c|c|c|c|c|}
\hline Date & Site Name & ${ }^{3} \mathrm{H}$ & noble gases & ${ }^{6} \mathrm{Cl}$ & ${ }^{14} \mathrm{C}$ & stable isotopes & ${ }^{87} \mathrm{Sr} /{ }^{86} \mathrm{Sr}$ & misc & 1291 \\
\hline & & & & & & & & & \\
\hline \multicolumn{10}{|c|}{$\frac{1}{\text { Pahranagat Valley }}$} \\
\hline $8 / 7 / 95$ & Issac Spencer irrigation well & $\mathrm{x}$ & $\bar{x}$ & $\bar{x}$ & $x$ & $x$ & $\bar{x}$ & $x$ & \\
\hline $8 / 7 / 95$ & Hiko Spring & $\mathrm{X}$ & $x$ & $\bar{x}$ & $\bar{x}$ & $\bar{x}$ & $\bar{x}$ & $\mathrm{x}$ & $\bar{x}$ \\
\hline $8 / 7 / 95$ & Crystal Spring. & $\mathrm{x}$ & $\bar{x}$ & $x$ & $\bar{x}$ & $\bar{x}$ & $\bar{x}$ & $\bar{x}$ & $\bar{x}$ \\
\hline $8 / 8 / 95$ & Alamo City well \#7 & $\mathrm{X}$ & $x$ & $\underline{x}$ & $\underline{x}$ & $\mathrm{x}$ & $\mathrm{x}$ & $x$ & \\
\hline $8 / 8 / 95$ & US Fish and Wildlife irrigation well & $x$ & $x$ & $\bar{x}$ & $\bar{x}$ & $x$ & $x$ & $x$ & \\
\hline $8 / 8 / 95$ & Sixmile Spring & $\mathrm{X}$ & & $\bar{x}$ & $x$ & $\bar{x}$ & $x$ & $x$ & \\
\hline $8 / 8 / 95$ & Little Ash Spring & $\mathrm{X}$ & $\bar{x}$ & $x$ & $x$ & $x$ & $\underline{x}$ & $x$ & $\underline{x}$ \\
\hline $8 / 8 / 95$ & Ash Spring & & & & $\bar{x}$ & $\bar{x}$ & & & \\
\hline $8 / 9 / 95$ & Stewart Brothers irrigation well & $\mathrm{x}$ & $\bar{x}$ & $\bar{x}$ & $\bar{x}$ & $x$ & $\bar{x}$ & $x$ & \\
\hline $8 / 9 / 95$ & Oreana Spring & $x$ & & $\bar{x}$ & $\bar{x}$ & $\underline{x}$ & $\bar{x}$ & $x$ & \\
\hline \multicolumn{10}{|c|}{ Emigrant Valley } \\
\hline $8 / 15-16 / 95$ & Watertown Well \#1 & \begin{tabular}{|l|}
$x$ \\
\end{tabular} & $\bar{x}$ & $\bar{x}$ & $\bar{x}$ & $\bar{x}$ & $\mathrm{x}$ & $\bar{x}$ & $x$ \\
\hline $8 / 15-16 / 95$ & Watertown Well \#3 & $\mathrm{X}$ & $x$ & $x$ & $\bar{x}$ & $\underline{x}$ & $\bar{x}$ & $x$ & $x$ \\
\hline $8 / 15-16 / 95$ & Watertown Well \#4 & $x$ & $\bar{x}$ & $x$ & $\bar{x}$ & $\bar{x}$ & $\bar{x}$ & $\bar{x}$ & $x$ \\
\hline & & & & & & & & & \\
\hline \multicolumn{10}{|l|}{ Ash Meadows } \\
\hline $9 / 6 / 95$ & Crystal Pool Spring & $\mathrm{x}$ & $x$ & $x$ & $x$ & $x$ & $x$ & $x$ & $x$ \\
\hline $9 / 6 / 95$ & Big Spring & $x$ & $x$ & $\bar{x}$ & $\bar{x}$ & $x$ & $\bar{x}$ & $x$ & $x$ \\
\hline $9 / 6 / 95$ & Fairbanks Spring & $\mathrm{X}$ & $\bar{x}$ & $\bar{x}$ & $\bar{x}$ & $\bar{x}$ & $x$ & $\bar{x}$ & $x$ \\
\hline \multicolumn{10}{|l|}{ Oasis Valley } \\
\hline $9 / 7 / 95$ & Goss Spring & \begin{tabular}{|l|}
$x$ \\
\end{tabular} & $\underline{x}$ & $\bar{x}$ & $x$ & $x$ & $\bar{x}$ & $x$ & $\bar{x}$ \\
\hline $9 / 7 / 95$ & Bailey Hot Spring & $x$ & $\underline{x}$ & $\bar{x}$ & $\bar{x}$ & $\bar{x}$ & $\bar{x}$ & $\bar{x}$ & $x$ \\
\hline $9 / 7 / 95$ & Revert Spring & $\mathrm{X}$ & $\bar{x}$ & $\bar{x}$ & $\underline{x}$ & $x$ & $x$ & $x$ & $\underline{x}$ \\
\hline & & & & & & & & & \\
\hline & & & & & & & & & \\
\hline Total number & of $s e$ & 18 & 16 & 18 & 19 & 19 & 18 & 18 & 12 \\
\hline
\end{tabular}




\section{SITE SELECTION}

The Las Vegas Office of the Water Resource Division of the USGS maintains an extensive database compiling the location, sample type, static water level and ownership of wells and springs in southern Nevada. This database served as the primary source of information for well and spring locations. Additional published material (Perfect et al., 1995, McKinley et al., 1991, Clary et al., 1994, as well as a regional geochemical database compiled by GeoTrans, 1994) and unpublished information (personal communications with DRI and USGS personnel) augmented the USGS database.

Sampling sites were chosen to maximize representative coverage of each area with concessions to allow for efficient sampling. Wells are more abundant in populated areas, and were carefully selected based on geographic location, perforation depths and usage. Irrigation wells were preferred over domestic supply wells because of the potential presence of chlorine and anti-microbial agents in the latter. In addition, it is likely that irrigation wells are more thoroughly purged than domestic wells. Several sites selected for sampling were unavailable due to well obstructions, inoperable pumps down hole, location on private property, erroneously mapped coordinates or dry holes. A hand-held global positioning system facilitated well location in remote or poorly/inaccurately mapped areas.

\section{Pahranagat Valley}

Hiko, Crystal and Ash springs are all high volume discharge springs (up to $0.51 \mathrm{~m}^{3} / \mathrm{s}$ ) located approximately $60 \mathrm{~km}$ northeast of NTS. Current. hypotheses suggest these springs discharge from the regional lower carbonate aquifer. Groundwater produced from private wells within Pahranagat Valley was also sampled to investigate recharge to this aquifer.

Springs discharging from Tertiary volcanic rocks in and around Pahranagat Valley are of interest to characterize recharge to local flow systems. Sixmile and Oreana Springs are perennial springs that discharge from nearby volcanic rocks and were chosen for their copious discharge $\left(\sim 3.3 \times 10^{-5} \mathrm{~m}^{3} / \mathrm{s}\right)$ and geographic location north and east of Pahranagat Valley.

\section{Emigrant Valley}

Several wells and springs present in the Emigrant Valley, located adjacent to the northeastern boundary of the NTS, are immediately upgradient of the NTS. Due to security restrictions, the LLNL HRMP sampling team was denied access to sites within this controlled area. However, LLNL trained and equipped authorized personnel from DRI to collect samples on our behalf. DRI personnel sampled three accessible water supply wells for LLNL (Watertown Well \#1, \#3 and \#4). No springs were sampled. 


\section{Ash Meadows}

Springs in the Ash Meadows discharge groundwater which originates in the Pahranagat Valley and eastern NTS (Waddell et al., 1984). As such, these waters incorporate isotope and chemical signatures indicative of regional groundwater flow paths and flow rates (Kenneally et al., 1995). Some of the springs are little more than seeps whose water chemistry may be affected by evaporation. To minimize the effects of isotope fractionation due to evaporation, LLNL chose three springs (Crystal Pool Spring, Big Spring and Fairbanks Spring) with the highest discharge rates. In addition, these springs are approximately equidistant along the $15 \mathrm{~km}$ long spring line in the Ash Meadows discharge area.

\section{Oasis Valley}

The discharge from some of the springs in Oasis Valley is associated with flow beneath Pahute Mesa. Like Ash Meadows, many of the springs in Oasis Valley are little more than seeps supporting abundant phreatophytes. We sampled three springs (Goss Spring, Bailey Hot Spring and Revert Spring) that exhibited minimal variance in discharge temperature and / or volume.

\section{North of the NTS}

Due to security restrictions, it was not possible to obtain access to U.S. Air Force lands north of the NTS for groundwater sampling during FY95.

\section{FY96 ACTIVITIES}

During FY96 LLNL will complete analysis of ${ }^{2} \mathrm{H} /{ }^{1} \mathrm{H},{ }^{3} \mathrm{H},{ }^{18} \mathrm{O} /{ }^{16} \mathrm{O},{ }^{13} \mathrm{C},{ }^{14} \mathrm{C}$, ${ }^{36} \mathrm{Cl},{ }^{129} \mathrm{I}$, noble gases, major cations and anions for the samples collected in FY95. These analyses will expand our existing isotope data-base beyond the confines of the Nevada Test Site and allow us to develop regional flow models for the Oasis Valley, Alkali Flat - Furnace Creek and Ash Meadows sub-basins of the Death Valley groundwater basin which includes the NTS. In addition, we will pursue opportunities with the USAF to sample in restricted areas north of the NTS closed to us in FY95. Ground and surface waters from Kawich Valley, Gold Flat, Reveille Valley and Cactus Flat, all north of the NTS, potentially recharge the Oasis Valley and Alkali Flat Furnace Creek groundwater sub-basins including the northern NTS and are important to our regional investigation. 


\title{
II. OPERATIONAL SUPPORT
}

\section{FY93, FY94 AND REPRESENTATIVE OLDER HOT WELL DATA}

\author{
Bradley K. Esser, Jacqueline M. Kenneally, Jo Ann H. Rego, \\ Jennifer L. Royval, David K. Smith
}

\section{INTRODUCTION}

Studies of radionuclide migration at the NTS rely on a measure of radionuclides dissolved in or potentially transported by groundwater. LLNL and LANL have collected and analyzed fluids returned from saturated underground nuclear test cavities to measure the concentration of radionuclides dissolved in groundwater. These efforts are part of a groundwater monitoring program for the NTS administered by the HRMP and predecessor programs from the mid-1970s. The following is a compilation of data collected in FY93 and FY94 as well as representative historical data from the 1970s and 1980s. The historical analyses are not a comprehensive list of all cavity fluids ever analyzed but rather analyses that LLNL has been able to verify through archived field and analytical records.

Figure 2 locates the eleven saturated nuclear event cavities for which we report data.

For the purposes of this compilation, wells are defined as impacted or 'hot' if the sampled cavity fluids contain radionuclides above the Nevada safe drinking water standard or were derived from a saturated cavity. In the state of Nevada, the drinking water standard for tritium $\left({ }^{3} \mathrm{H}\right)$ is $20,000 \mathrm{pCi} / \mathrm{L}$. To facilitate comparison, all radionuclide data have been decay corrected to January 1,1994. This date also corresponds to that selected for reporting an event-by-event and cumulative inventory of residual tritium, fission products, activation products and actinides for all underground nuclear tests fired at the NTS (Goishi et al., 1994).

Less than twenty of over approximately 150 saturated cavities at the Nevada Test Site are available for sampling. Because cavity wells are not constructed, completed or produced as are conventional water supply wells, sampled fluids may not be representative of true radionuclide concentrations dissolved within the cavity. As a result, the reported data must be evaluated relative to the occurrence of radionuclides in an individual event cavity, construction of the well and means of producing the sample.

Table II includes data returned during FY94; Table III includes data returned in FY93 and Table IV is a compilation of the older historical data. 


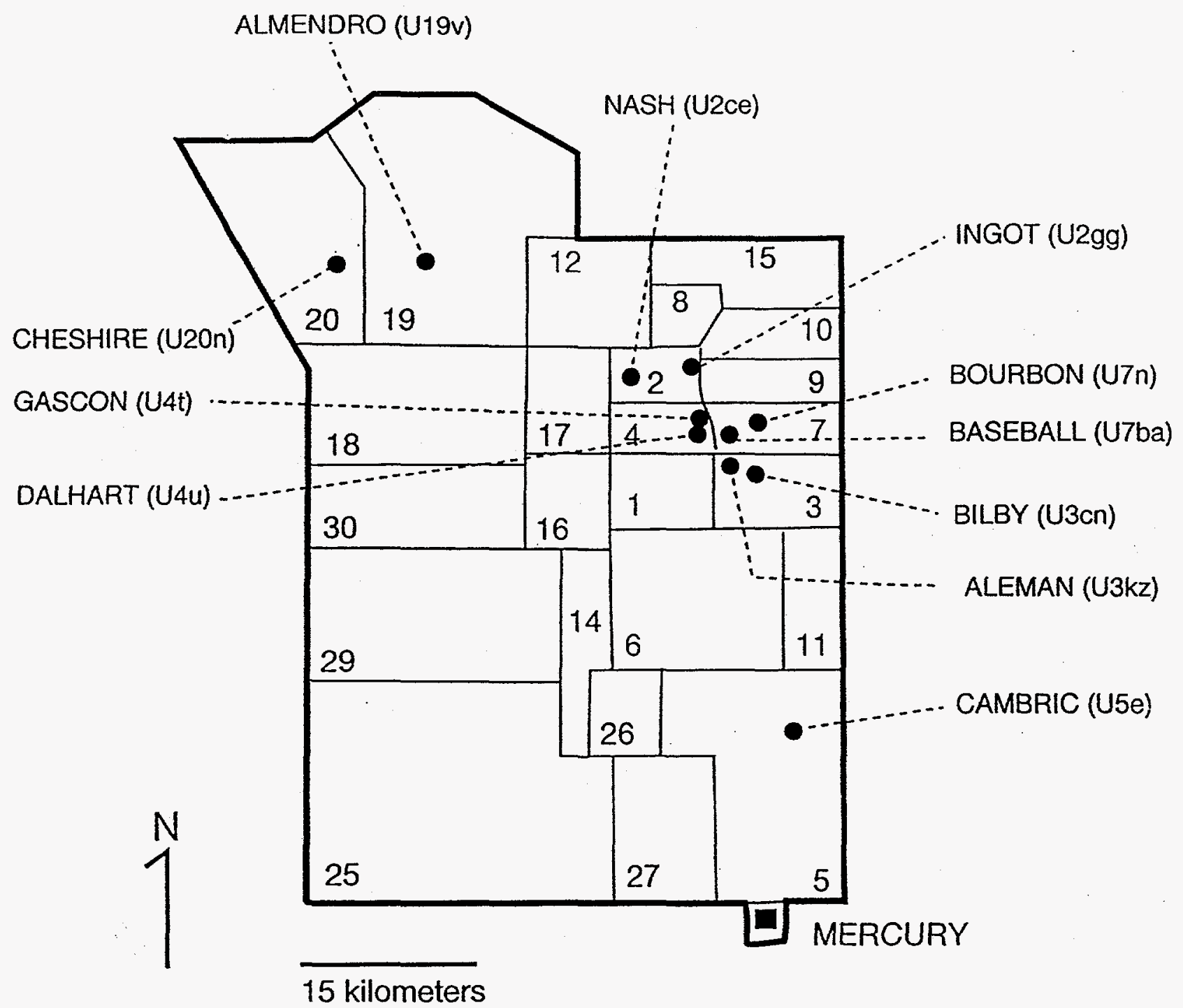

Figure 2: Location of underground nuclear test events with saturated cavities referenced in this report. 


\section{DATA QUALIFICATIONS}

Because there are differences in sample collection, analysis and interpretation for contaminated and uncontaminated waters at the NTS, explanatory statements preface the following data tables. The Isotope Sciences Division of LLNL maintains a field log of 'hot' well sampling tours conducted in FY93, FY94 and FY95. Older records are less comprehensive but were assembled and verified by personnel historically involved with that sampling. As noted above, event phenomenology, well construction and sampling artifacts combine to compromise collection of a representative sample. Specific comments follow:

- The phenomenology of underground nuclear explosions results in a heterogeneous distribution of radionuclides in the subsurface cavity and overlying chimney. The distribution of radionuclides produced during an underground nuclear explosion is discussed by Thompson (1995) and Smith (1995) and will not be repeated here. Because the residual radionuclides are highly fractionated and not equally available for transport, the resulting dissolved concentrations are nonuniform. The transfer of radionuclides from a solid to a soluble residual is controlled by release functions which are presently only poorly understood (Smith et al., 1995).

- An analysis of a single well does not represent an average for all cavity fluịds. Radionuclide yields and distributions are device specific. For example, $16 \%$ of the events fired below the static water level on the Pahute Mesa produced $70 \%$ of tritium for that area (Goishi et al., 1994).

- No effort was made to remove drilling fluid, cement or other additives introduced during drilling and completion cavity or near-cavity wells. Post-shot drilling procedure is to shut-in the hole at the first indication of radioactivity and inject drilling muds directly to the formation with no surface returns. Bentonite clays and colloids present in the muds will sorb radionuclides; in addition, bentonite based drilling muds have high levels of naturally occurring radionuclides. Esser (1994) reports the Area 3 NTS drilling mud contains $4.0 \mathrm{pCi} / \mathrm{g}$ of $40 \mathrm{~K}, 2.1 \mathrm{pCi} / \mathrm{g}$ of ${ }^{232} \mathrm{Th}$ and $2.8 \mathrm{pCi} / \mathrm{g}$ of 238 U. Although these isotopes are distinct from fission and activation products and actinides produced during an underground nuclear explosion, these nuclides augment concentrations of natural occurring radionuclides incorporated in nuclear explosive melt debris.

- As indicated in the tables a majority of the samples were collected using evacuated 2 liter bailers. Pumps were used in a minority of wells (CHESHIRE (U20n ps1ddh), CAMBRIC (RNM-1, RNM-2S), BILBY (U3cn, U3cn5)); however, the majority of wells consist of a single carbon steel piezometer access tube without a pump completed in or adjacent to the 
cavity. Fluids trapped in an open piezometer tube will not circulate. The effects of NTS well construction and development techniques on hydrochemical and radionuclide indicators is described in a draft DOE report and will not be repeated here (DOE, 1994). Graham and Johnson (1991) indicate well development ratios (volume of fluid removed divided by the volume of fluid lost during drilling) in excess of 100 is required for 1000 fold reduction in conservative drilling fluid tracers. A ratio of greater than 1000 may be required for conservative isotopes. The use of bailers precludes well development. Finally, raising and lowering a bailer in an open piezometer tube creates turbulence in the water column and affects reproducible sampling.

- Sample filtration and acidification pre-treatments are variable. Data tables indicate which samples were filtered and through which nominal pore size filter. Buddemeier and Hunt (1988) emphasize transport of radionuclides on particles and colloids smaller than $0.45 \mu \mathrm{m}$ break which conventionally defines dissolved species. Filtration will obviously remove radionuclides associated with suspended particles and colloids. Some of the fluids were acidified to prevent precipitation of equilibrium phases which would alter the water chemistry. Shifts in $\mathrm{pH}$ and Eh may affect the concentration of dissolved radionuclides with multiple oxidation states (i.e, $\mathrm{U}, \mathrm{Tc}, \mathrm{Pu}, \mathrm{Np}$ ).

- Results for gross beta analyses do not include all beta emitting species. Sample preparation for gross beta counting include digestion and evaporation that removes both ${ }^{3} \mathrm{H}$ and ${ }^{14} \mathrm{C}$ from the sample. Similarly ${ }^{85} \mathrm{Kr}$, a gaseous beta emitter, is not included in gross beta measurements.

- LANL and LLNL jointly sampled adjacent to the INGOT cavity in FY94. LANL data are included in this tabulation and the source is noted.

- FY93 samples were submitted to IT Corporation for analysis of volatile and semi-volatile compounds. Samples with analyte concentrations above detectable limits are included in Table III.

- The field parameters and major and trace elements reported for CHESHIRE (U20nPS\#1) represents an average of discrete samples collected during high volume ( $10^{5}$ to $10^{7}$ liters) pumping of the CHESHIRE cavity. Sampling information and data is derived from Buddemeier and Isherwood (1985) and Buddemeier and Hunt (1988).

- The designation "p1, p2, p3" for the ALEMAN (UE3e\#4) well refers to individual nested piezometers within a single bore. 


\section{DESCRIPTION OF THE DATA TABLES}

- Well name, corresponding event name, means of collection (pumped or bailed with depth of collection) and sampling date are listed together with the analyte in the header for each table. Some wells were slant drilled and completed at an inclined angle. In all cases collection depths for the bailed samples represent measured distance from the surface down the well casing and not vertical depth below surface.

- Concentration and activity units are presented in the second row of each column.

- Radionuclide half-lives, in years, are given in the third row of each column.

- Radionuclide data have been decay corrected to both the date of collection and January 1, 1994 and are listed beneath the header for row four.

- Where data are not available, data fields contain a dash (-).

- Data in concentrations or activities less than the limit of analytical detection are indicated by an inequality $(<)$. 
TABLE II

\begin{tabular}{|c|c|c|c|c|c|c|c|c|c|c|c|c|c|}
\hline Well Name & Event & Depth & Lab & $\begin{array}{l}\text { Collection } \\
\text { Date }\end{array}$ & DO & $\mathrm{pH}$ & $\mathrm{T}$ & $\mathrm{EC}$ & $\begin{array}{l}\text { Gross } \\
\text { alpha }\end{array}$ & $\begin{array}{l}\text { Gross } \\
\text { beta }\end{array}$ & $3 \mathrm{H}$ & $3 \mathrm{H}$ & $14 \mathrm{C}$ \\
\hline Units & & $(f t)$ & & & $(m g / L)$ & & $\left({ }^{\circ} \mathrm{C}\right)$ & $(\mu s)$ & $(p C i / L)$ & $(p \mathrm{Ci} / \mathrm{L})$ & $(p C i / L)$ & $(p C i / L)$ & $(p C i / L)$ \\
\hline Half-life (a) & & & & & & & & & & & 12.3 & 12.3 & 5730 \\
\hline Reference date & & & & & & & & & collect. & collect. & collect. & $1 / 1 / 94$ & collect. \\
\hline Field blank & - & & LLNL & $9 / 12 / 94$ & - & - & - & - & $<1.40 \mathrm{e}+0$ & $<2.20 \mathrm{e}+0$ & & & $<3.00 \mathrm{e}+2$ \\
\hline U2gg psE3a & Ingot & 1927 & LLNL & $9 / 22 / 94$ & - & - & - & - & & $3.45 e+1$ & $4.00 \mathrm{e}+3$ & $4.17 e+3$ & $2.00 \mathrm{e}+4$ \\
\hline U2gg psE3a & Ingot & 1960 & LLNL & $9 / 22 / 94$ & $1-2$ & 7.47 & 26.0 & 2280 & $1.26 \mathrm{e}+0$ & $<1.68 \mathrm{e}+1$ & $4.00 \mathrm{e}+3$ & $4.17 e+3$ & $6.00 \mathrm{e}+3$ \\
\hline U2gg psE3a & Ingot & 1960 & LANL & $9 / 22 / 94$ & - & - & - & - & - & - & $7.80 \mathrm{e}+3$ & $8.12 \mathrm{e}+3$ & \\
\hline U20n ps1ddh & Cheshire & 2248 & LLNL & $9 / 14 / 94$ & 2.0 & 8.35 & 27.8 & 386 & - & $8.85 e+1$ & $6.50 \mathrm{e}+7$ & $6.76 e+7$ & $5.30 \mathrm{e}+4$ \\
\hline U20n pslddh & Cheshire & 2448 & LLNL & $9 / 14 / 94$ & - & $=$ & $=$ & - & - & - & $6.50 \mathrm{e}+7$ & $6.76 \mathrm{e}+7$ & \\
\hline U20n ps1ddh & Cheshire & 2648 & LLNL & $9 / 14 / 94$ & - & - & 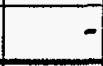 & - & - & $7.71 \mathrm{e}+1$ & $6.50 \mathrm{e}+7$ & $6.76 e+7$ & $6.10 e+4$ \\
\hline $\begin{array}{l}\text { NOTE: } \\
\text { Detection } \\
\text { limit }=<\end{array}$ & & & & & & & & & & & & & \\
\hline
\end{tabular}




\begin{tabular}{|c|c|c|c|c|c|c|c|c|c|}
\hline Well Name & $85 \mathrm{Kr}$ & $85 \mathrm{Kr}$ & $125 \mathrm{Sb}$ & $125 \mathrm{Sb}$ & $137 \mathrm{Cs}$ & $137 \mathrm{Cs}$ & $238 \mathrm{Pu}$ & $238 \mathrm{Pu}$ & $239,240 \mathrm{Pu}$ \\
\hline Units & $(p C i / L)$ & $(p C i / L)$ & $(p C i / L)$ & $(p C i / L)$ & $\overline{(p C i / L)}$ & $(p C i / L)$ & $\overline{(p C i / L)}$ & $\overline{(p C i / L)}$ & $(p C i / L)$ \\
\hline Half-life (a) & 10.7 & 10.7 & 2.8 & 2.8 & 30.2 & 30.2 & 87.7 & 87.7 & $\geq 6500$ \\
\hline Reference date & collect. & $1 / 1 / 94$ & collect. & $1 / 1 / 94$ & collect. & $1 / 1 / 94$ & collect. & $1 / 1 / 94$ & collect. \\
\hline Field blank & . & . & $<6.00 \mathrm{e}-1$ & $<7.15 \mathrm{e}-1$ & $<2.50 \mathrm{e}-1$ & $<2.54 \mathrm{e}-1$ & $2.60 \mathrm{e}-9$ & $2.61 e-9$ & \\
\hline U2gg psE3a & - & & $<9.60 \mathrm{e}-1$ & $<1.15 \mathrm{e}+0$ & $9.00 e-1$ & $9.15 e-1$ & & & \\
\hline U2gg psE3a & & & $<1.20 \mathrm{e}+0$ & $<1.44 \mathrm{e}+0$ & $2.70 \mathrm{e}+0$ & $2.75 e+0$ & $2.00 \mathrm{e}-9$ & $2.01 e-9$ & \\
\hline U2gg psE3a & $1.00 \mathrm{e}+1$ & $1.05 e+1$ & - & - & & & & & \\
\hline U20n psiddh & - & 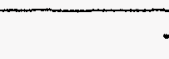 & $<1.00 \mathrm{e}+0$ & $<1.19 \mathrm{e}+0$ & $2.30 \mathrm{e}+1$ & $2.34 \mathrm{e}+1$ & $2.40 e-8$ & $2.41 \mathrm{e}-8$ & $8.60 \mathrm{e}-8$ \\
\hline U20n ps1ddh & - & . & $<9.50 \mathrm{e}-1$ & $<1.13 \mathrm{e}+0$ & $2.30 e+1$ & $2.34 e+1$ & - & & \\
\hline U20n ps1ddh & - & . & $6.70 \mathrm{e}+0$ & $7.99 \mathrm{e}+0$ & $3.40 \mathrm{e}+1$ & $3.46 \mathrm{e}+1$ & $4.20 e-9$ & $4.22 \mathrm{e}-9$ & $7.60 \mathrm{e}-9$ \\
\hline $\begin{array}{l}\text { NOTE: } \\
\text { Detection } \\
\text { limit }=<\end{array}$ & & & & & & & & & \\
\hline
\end{tabular}




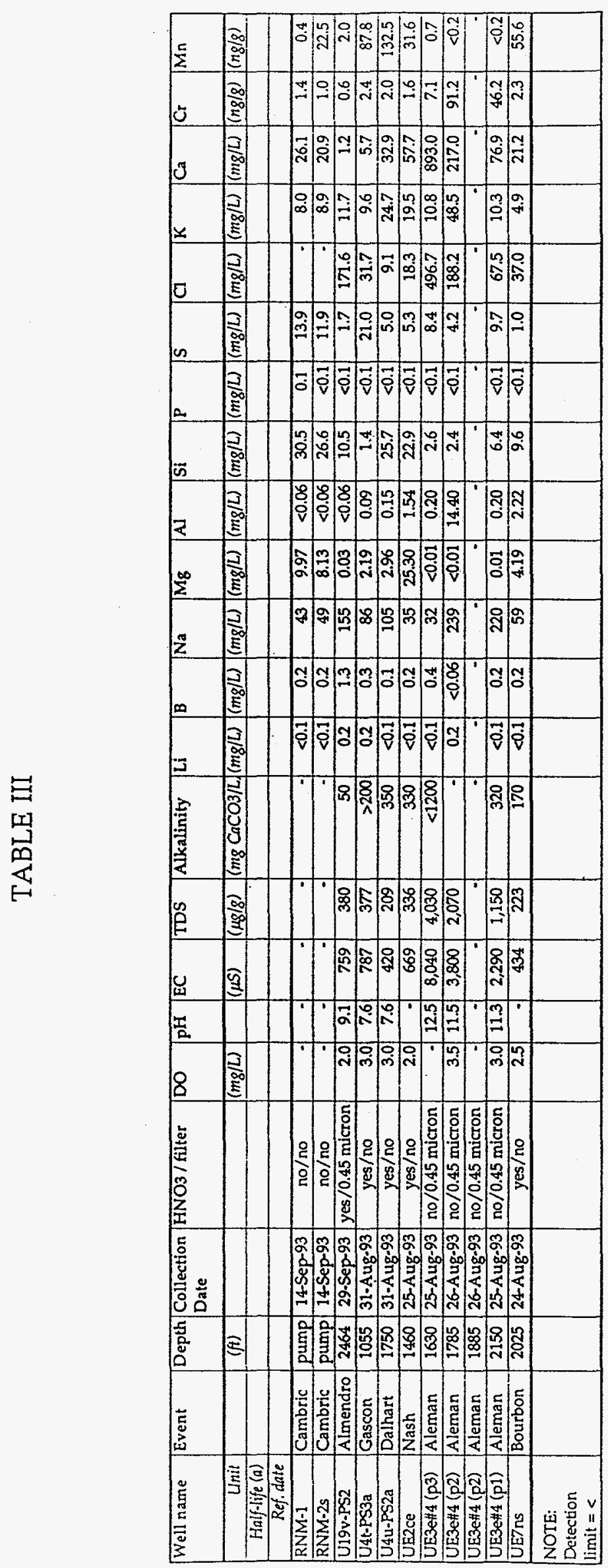




\begin{tabular}{|c|c|c|c|c|c|c|c|c|c|c|c|c|c|c|c|c|c|c|c|c|c|}
\hline Well name & $\mathrm{Fe}$ & $C_{0}$ & $\mathrm{Ni}$ & $\mathrm{Cu}$ & $\overline{Z n}$ & As & Se & $\mathrm{Sx}$ & Mo & $\overline{\mathbf{B a}}$ & $\overline{\mathrm{Hg}_{\mathrm{B}}}$ & $\mathrm{Pb}$ & $\bar{u}$ & $\begin{array}{l}\text { Gross } \\
\text { alpha }\end{array}$ & $\begin{array}{l}\text { Gross } \\
\text { beta }\end{array}$ & $3 \mathrm{H}$ & $3 \mathbf{H}$ & $60 \mathrm{Co}$ & $60 \mathrm{Co}$ & $125 \mathrm{Sb}$ & $125 \mathrm{Sb}$ \\
\hline Unit & $(m g / L)$ & $(n g / g)$ & $(n g / g)$ & $(n g / g)$ & $(n g / g)$ & $(n g / g)$ & $(n g / g)$ & $(\mathrm{ng} / \mathrm{g})$ & $(n g / g)$ & $(n g / g)$ & $(n g / g)$ & $(\mathrm{ng} / \mathrm{g})$ & $(\pi g / 8)$ & $(\mathrm{pCl} / \mathrm{L})$ & $(p C i / L)$ & $(p C i / L)$ & $(p C i / L)$ & $(p C i / L)$ & (pCi/L) & $(p C i / L)$ & $(p C i / L)$ \\
\hline Half-life (a) & & & & & & & & & & & & & & & & 12.3 & 12.3 & 5.3 & 5.3 & 2.8 & 2.8 \\
\hline Ref. date & & & & & & & & & & & & & & collect. & collect. & collect. & $1 / 1 / 94$ & collect. & $1 / 1 / 94$ & collect. & $1 / 1 / 94$ \\
\hline RNM-1 & 0.03 & $<0.1$ & $<0.1$ & 1.1 & 8.0 & 7.0 & 8.4 & 266 & 3.7 & 14.8 & 1.1 & 0.8 & 3.9 & $<4.81 \mathrm{e}-2$ & $<9.62 \mathrm{e}-2$ & $<2.00 \mathrm{e}+2$ & $<1.97 \mathrm{e}+2$ & $<4.44 e-4$ & $<4.27 \mathrm{e}-4$ & $<1.33 \mathrm{e}-3$ & $<1.24 \mathrm{e}-3$ \\
\hline RNM-2s & 0.06 & $<0.1$ & $<0.1$ & 1.0 & 2.0 & 5.0 & 9.5 & 120 & 4.1 & 24.1 & 1.0 & 1.4 & 3.5 & $<4.81 \mathrm{e}-2$ & $<9.62 \mathrm{e}-2$ & $2.60 \mathrm{e}+5$ & $2.56 e+5$ & $<3.07 \mathrm{e}-4$ & $<2.95 \mathrm{e}-4$ & $<9.25 \mathrm{e}-4$ & $<8.58 \mathrm{e}-4$ \\
\hline U19v-PS2 & 0.12 & $<0.1$ & $<0.1$ & 1.5 & 24.0 & 105.0 & 23.0 & 47 & $1,007.0$ & 49.2 & 2.0 & 0.8 & $<0.1$ & $<4.81 \mathrm{e}-2$ & $<9.62 \mathrm{e}-2$ & $2.30 \mathrm{e}+8$ & $2.27 \mathrm{e}+8$ & $<4.442-4$ & $<4.29 e-4$ & $<1.15 \mathrm{e}-3$ & $<1.08 \mathrm{e}-3$ \\
\hline U4t-PS3a & 4.01 & 0.4 & 24.0 & 31.2 & $3,323.0$ & 5.8 & 29.0 & 963 & 111.4 & 127.0 & $<0.5$ & 194.0 & 0.3 & $<4.81 \mathrm{e}-2$ & $<9.62 \mathrm{e}-2$ & $6.70 \mathrm{e}+4$ & $6.57 \mathrm{e}+4$ & $<4,44$ & $<4.25 \mathrm{e}-4$ & $<1.18 \mathrm{e}-3$ & $<1.09 \mathrm{e}-3$ \\
\hline U4u-PS2a & 1.58 & 0.3 & 26.7 & 63.1 & 409.7 & 9.5 & 16.9 & 46 & 1.6 & 9.0 & 1.0 & 4.4 & 6.3 & $<4.81 \mathrm{e}-2$ & $<9.62 \mathrm{e}-2$ & $4.92 e+7$ & $4.83 e+7$ & $1.40 \mathrm{e}-2$ & $1.34 e-2$ & $2.08 \mathrm{e}+0$ & $1.91 \mathrm{e}+0$ \\
\hline UE2ce & 0.90 & $<0.1$ & $<0.1$ & 3.2 & 314.7 & 1.6 & 5.5 & 217 & 14.2 & 29.5 & $<0.5$ & 23.5 & 0.4 & $<4.81 \mathrm{e}-2$ & $<9.62 \mathrm{e}-2$ & $1.30 \mathrm{e}+5$ & $1.27 e+5$ & $<4.07 e-4$ & $<3.89 \mathrm{e}-4$ & $<1.18 \mathrm{e}-3$ & $<1.08 \mathrm{e}^{-3}$ \\
\hline UE3e\#4 (p3) & $<0.02$ & 10.8 & 71.0 & 190.3 & 585.4 & 194.0 & 65.0 & 8,084 & 28.7 & $1,140.0$ & 9.1 & 856.0 & $<0.1$ & $<4.81 \mathrm{e}-2$ & $<9.62 \mathrm{e}-2$ & $4.39 \mathrm{e}+5$ & $4.30 e+5$ & $<5.92 \mathrm{e}-4$ & $<5.65 \mathrm{e}-4$ & $<1.74 \mathrm{e}-3$ & $<1.59 \mathrm{e}-3$ \\
\hline UE3e\#4 (p2) & $<0.02$ & 1.0 & 1.1 & 40.4 & 117.9 & 14.9 & 73.4 & 4,471 & 26.4 & $1,518.0$ & 3.1 & 8.5 & $<0.1$ & $<4.81 \mathrm{e}-2$ & $<9.62 \mathrm{e}-2$ & $4.77 \mathrm{e}+4$ & $4.68 \mathrm{e}+4$ & & & & \\
\hline UE3e\#4 (p2) & & & & & & & & $\square$ & & & & & & $<4.81 \mathrm{e}-2$ & $9.62 \mathrm{e}-2$ & $3.30 \mathrm{e}+5$ & $3.24 \mathrm{e}+5$ & -1 & & & \\
\hline UE3e\#4 (p1) & $<0.02$ & 0.4 & $<0.1$ & 60.3 & 6.4 & 7.2 & 30.7 & 487 & 41.8 & 144.0 & 1.1 & 18.0 & $<0.1$ & $<4.81 e-2$ & $<9.62 e-2$ & $9.42 e+6$ & $9.23 e+6$ & & & & \\
\hline UE7ns & 0.28 & $<0.1$ & $<0.1$ & 8.8 & 234.0 & 4.8 & 8.1 & 144 & 36.4 & 125.0 & $<0.5$ & 15.0 & $<0.1$ & $<4.81 \mathrm{e}-2$ & $<9.62 \mathrm{e}-2$ & $4.57 \mathrm{e}+2$ & $4.48 \mathrm{e}+2$ & $<3.33 \mathrm{e}-4$ & $<3.18 \mathrm{e}-4$ & $<8.14 e^{-4}$ & $<7.44-4$ \\
\hline $\begin{array}{l}\text { NOTE: } \\
\text { Detection } \\
\text { limit }=<\end{array}$ & & & & & & & & & & & & & & & & & & & & & \\
\hline
\end{tabular}




\begin{tabular}{|c|c|c|c|c|c|c|c|c|c|c|c|c|c|c|c|c|}
\hline Well name & $137 \mathrm{Cs}$ & $137 \mathrm{Cs}$ & 226Ra & $239 \mathrm{Pu}$ & $240 \mathrm{Pu}$ & \begin{tabular}{|l|} 
Carbon \\
disulfide
\end{tabular} & $\begin{array}{l}\text { TCE } \\
1,1,1\end{array}$ & Benzene & \begin{tabular}{|l|} 
Methyl \\
chloride
\end{tabular} & Toluene & 2-butanone & $\begin{array}{l}\text { Dimethyl } \\
\text { ether }\end{array}$ & Phenol & $\begin{array}{l}\text { 2-Methyl } \\
\text { phenol }\end{array}$ & $\begin{array}{l}\text { 4-Methyl } \\
\text { phenol }\end{array}$ & $\begin{array}{l}\text { Di-n-butyl } \\
\text { phthalate }\end{array}$ \\
\hline Unit & $(p C i / L)$ & $(p \subset i / L)$ & $(p C i / L)$ & $(p C i / L)$ & $(p C i / L)$ & $(\mu g / L)$ & $(\mu g / L)$ & $(\mu g / L)$ & $(\mu g / L)$ & $(\mu g / L)$ & $(\mu g / L)$ & $(\mu g / L)$ & $(\mu \mathrm{g} / L)$ & $(\mu g / L)$ & $(\mu g / L)$ & $(\mu g / L)$ \\
\hline Half-life (a) & 30.2 & 30.2 & 1600 & 24100 & 6560 & & & & & & & & & & & \\
\hline Ref. date & collect. & $1 / 1 / 94$ & collect. & collect. & collect. & & & & & & & & & & & \\
\hline RNM-1 & $9.25 \mathrm{e}-2$ & $9.19 e-2$ & $<2.73 e-2$ & $<8.88 \mathrm{e}-5$ & $<7.40 \mathrm{e}-5$ & 1.0 & 2.0 & 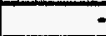 & 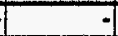 & - & 7 & 20 & & - & - & \\
\hline RNM-2s & $<3.29 \mathrm{e}-4$ & $<3.27 \mathrm{e}-4$ & $2.34 \mathrm{e}-3$ & $<1.48 \mathrm{e}-4$ & $<9.62 \mathrm{e}-5$ & 1.0 & 1.0 & 0.7 & 0.3 & 0.5 & 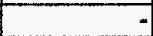 & 20 & & - & & \\
\hline U19v-PS2 & $<5.18 \mathrm{e}-4$ & $<5.15 \mathrm{e}-4$ & $<9.62 \mathrm{e}-4$ & $<3.15 \mathrm{e}-4$ & $<1.85 e-4$ & 0.4 & & 4.0 & $\because$ & -1 & 10 & +1 & 27 & 26 & 65 & 31 \\
\hline U4t-PS3a & $<4.81 \mathrm{e}-4$ & $<4.77 e-4$ & $<8.88 \mathrm{e}-4$ & $<1.15 \mathrm{e}-3$ & $<9.25 \mathrm{e}-4$ & 2.9 & 1.0 & 1.0 & -1 & 0.6 & - & 20 & - & -1 & 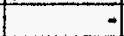 & - \\
\hline U4u-PS2a & $2.77 e-2$ & $2.75 e-2$ & $<8.88 \mathrm{e}-4$ & $<1.70 \mathrm{e}-4$ & $1.86 e-4$ & 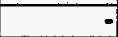 & 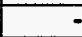 & - & -7 & - & - & 30 & - &. & - & t \\
\hline UE2ce & $<3.70 \mathrm{e}-4$ & $<3.67 \mathrm{e}-4$ & $8.88 e-3$ & $<1.18 \mathrm{e}-4$ & $<5.92 \mathrm{e}-5$ & - & - & 1.0 & - & -1 & - & 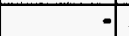 & - & 4 & - & \\
\hline UE3e\#4 (p3) & $<6.29 \mathrm{e}-4$ & $<6.24 e-4$ & $<1.30 e^{-3}$ & $<1.41 \mathrm{e}-4$ & $<1.18 \mathrm{e}-4$ & - & - & 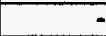 & -1 & - & - & -1 & 7 & - & 4 & \\
\hline UE3e\#4 (p2) & & & & $<1.48 \mathrm{e}-4$ & $<8.88 \mathrm{e}-5$ & - & & $=$ & - & 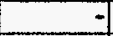 & - & & - & & - & \\
\hline UE3ell4 (p2) & & & & $<1.78 \mathrm{e}-4$ & $<1.07 \mathrm{e}-4$ & - & & $=$ & - & $t$ & - & - & -1 & - & $\exists$ & \\
\hline UE3 & 7 & & 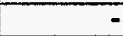 & $<1.07 e-4$ & $<1.04-4$ & - & $=$ & $=$ & - & 1 & 1 & - & 7 & 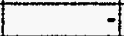 & - & \\
\hline UE7ns & $<3.44 \mathrm{e}-3$ & $<3.41 \mathrm{e}-3$ & $<7.03-4$ & $<9.25-5$ & $<9.99 \mathrm{e}-5$ & - & & - & 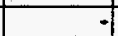 & - & 1 & 7 & -1 & - & 7 & \\
\hline $\begin{array}{l}\text { NOTE: } \\
\text { Detection } \\
\text { limit }=<\end{array}$ & & & & & & & & & & & & & & & & \\
\hline
\end{tabular}


TABLE IV

\begin{tabular}{|c|c|c|c|c|c|c|c|c|c|c|c|c|c|c|c|c|c|c|}
\hline Well name & Event & Collection date & Collection & HNO3/filter & pH & DO & TDS & $\mathbf{L i}$ & $\mathrm{Na}$ & $\mathrm{Mg}$ & C & $\mathbf{K}$ & $\mathrm{Ca}$ & Sr & Field 3H & Field 3H & $22 \mathrm{Na}$ & $22 \mathrm{Na}$ \\
\hline Unit & & & & & & $(m g / L)$ & $(m g / k g)$ & $(m g / L)$ & $(m g / L)$ & $(m g / L)$ & $(m g / L)$ & $(m g / L)$ & $(m g / L)$ & $(n g / g)$ & $(p C i / L)$ & $(p \mathrm{Ci} / \mathrm{L})$ & $(p \mathrm{Ci} / \mathrm{L})$ & $(p C i / L)$ \\
\hline Half-life $(a)$ & & & & & & & & & & & & & & & & 12.3 & & 2.605 \\
\hline Ref.date & & & & & & & & & & & & & & & collect. & $1 / 1194$ & collect. & $1 / 1 / 94$ \\
\hline $\mathrm{U} 3 \mathrm{cn}$ & Bilby & $9 / 27 / 77$ & pump & no/no & - & $\div$ & $\div$ & - & - & - & $=$ & $\div$ & - & $=$ & $1.72 e+8$ & $6.90 \mathrm{e}+7$ & $<4.44 \mathrm{e}-3$ & $<5.88 \mathrm{e}-5$ \\
\hline$\overline{U 3 \mathrm{cn}}$ & Bilby & $9 / 29 / 77$ & pump & no / no & - & - & - & - & - & 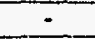 & - & $\therefore$ & $=$ & - & $1.76 \mathrm{e}+8$ & $7.04 \mathrm{e}+7$ & $<4.44 \mathrm{e}^{-3}$ & $<5.88 e^{-5}$ \\
\hline $\mathrm{U} 3 \mathrm{cn}$ & Bilby & $9 / 29 / 77$ & pump & no/no & - & - & - & - & $=$ & - & - & - & - & - & $1.80 e+8$ & $7.21 e+7$ & $<4.44 e^{-3}$ & $<5.88 \mathrm{e}-5$ \\
\hline$\overline{\mathrm{U} 3 \mathrm{Cn}}$ & Bilby & $9 / 29 / 77$ & pump. & no/no & - & - & - & - & - & - & - & - & - & - & $1.75 \mathrm{e}+8$ & $7.02 e+7$ & $<4.44 \mathrm{e}-3$ & $<5.88 \mathrm{e}-5$ \\
\hline $\mathrm{U} 3 \mathrm{cn}$ & Bilby & $9 / 29 / 77$ & pump & no/no & - & - & - & - & - & - & - & - & - & - & $1.68 \mathrm{e}+8$ & $6.74 \mathrm{e}+7$ & $<4.44 \mathrm{e}-3$ & $<5.88 \mathrm{e}-5$ \\
\hline $\mathrm{U} 3 \mathrm{cn}$ & Bilby & $9 / 29 / 77$ & pump & no/no & - & - & 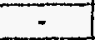 & - & - & $=$ & - & - & - & $\cdot$ & $1.74 \mathrm{e}+8$ & $6.96 \mathrm{e}+7$ & $<4.44 \mathrm{e}-3$ & $<5.88 \mathrm{e}-5$ \\
\hline U3en & Bilby & $9 / 29 / 77$ & pump & no/no & - & - & - & - & - & - & - & - & - & - & $1.72 \mathrm{e}+8$ & $6.87 \mathrm{e}+7$ & $<4.44 \mathrm{e}-3$ & $<5.88 \mathrm{e}-5$ \\
\hline $\mathrm{U} 3 \mathrm{cn}$ & Bilby & $9 / 30 / 77$ & pump & no/no & - & - & 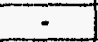 & - & - & - & - & - & - & 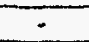 & $1.72 \mathrm{e}+8$ & $6.87 \mathrm{e}+7$ & $<4.44 \mathrm{e}-3$ & $<5.88 \mathrm{e}-5$ \\
\hline U3en & Bilby & $10 / 21 / 81$ & pump & no / no & - & - & 432 & - & - & - & $\therefore$ & - & $=$ & - & $1.81 \mathrm{e}+8$ & $7.23 e+7$ & $<4.44 e-3$ & $<5.88 \mathrm{e}-5$ \\
\hline $\mathrm{U} 3 \mathrm{~cm}$ & Bilby & $6 / 18 / 85$ & $\div$ & no/no & - & - & $\overline{341}$ & - & - & $\bullet$ & - & - & - & $\because$ & $2.08 e+8$ & $8.32 e+7$ & $<4.44 \mathrm{e}-3$ & $<5.88 \mathrm{e} 5$ \\
\hline U3en-n5 & Bilby & $10 / 21 / 80$ & pump & no $/ 1$ micron & - & - & 393 & - & - & - & - & - & $=$ & - & $4.33 e+1$ & $1.73 e+1$ & $<4.44 \mathrm{e}-3$ & $<5.88 \mathrm{e} 5$ \\
\hline U $3 \mathrm{cn}-\mathrm{n} 5$ & Bilby & $10 / 20 / 81$ & pump & no $/ 1$ micron & - & - & 304 & - & - & - & $\overline{-}$ & 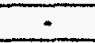 & - & - & $1.15 \mathrm{e}+1$ & $4.58 \mathrm{e}+0$ & $<4.44 \mathrm{e}-3$ & $<5.88 \mathrm{e} 5$ \\
\hline UE2ce & Nash & $3 / 15 / 78$ & pump & no $/ 1$ micron & 6.9 & $\div$ & 609 & 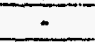 & 51 & 44 & 103 & 34 & 97 & 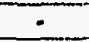 & $6.22 \mathrm{e}+7$ & $2.55 \mathrm{e}+7$ & $1.51 \mathrm{e}+0$ & $2.25 \mathrm{e}-2$ \\
\hline UE2ce & Nash & $10 / 9 / 80$ & pump & no $/ 1$ micron & - & - & 354 & $\therefore$ & $\therefore$ & $\therefore$ & - & $=$ & $=$ & - & $\therefore$ & $\therefore$ & $8.66 a-1$ & $1.29 \mathrm{e}-2$ \\
\hline UE2ce & Nash & $2 / 22 / 82$ & pump & no $/ 1$ micron & $=$ & - & 415 & 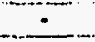 & - & $\because$ & $\div$ & - & - & $\because$ & $1.07 e+7$ & $437 e+6$ & $3.11 \mathrm{e}-1$ & $4.64 x-3$ \\
\hline UE2ce & Nash & $7 / 9 / 82$ & pump & no $/ 1$ micron & $=$ & - & 535 & - & - & - & - & $=$ & - & - & $9.77 \mathrm{e}+7$ & $4.01 e+7$ & $2.18 \mathrm{e}+0$ & $3.25 \mathrm{e}-2$ \\
\hline UE2ce & Nash & $8 / 10 / 83$ & pump & no $/ 0.45$ micron & 7.5 & - & 442 & - & 45.8 & 31.4 & 49.2 & 22.1 & 70.9 & 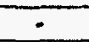 & $8.70 \mathrm{e}+7$ & $3.57 \mathrm{e}+7$ & $2.46 e+0$ & $3.68 \mathrm{e}-2$ \\
\hline UE2ce & Nash & $4 / 18 / 84$ & pump & no $/ 1$ micron & 6.6 & - & 567 & 0.17 & 62.7 & 39.9 & 108.8 & 22.3 & 94.7 & 0.3 & $1.62 e+8$ & $6.63 e+7$ & $4.37 \mathrm{e}+0$ & $6.53 \mathrm{e}-2$ \\
\hline UE2ce & Nash & $8 / 1 / 85$ & - & no / 1 micron & - & - & - & $\therefore$ & - & - & - & - & - & - & $1.26 \mathrm{e}+8$ & $5.16 e+7$ & $\div$ & $\cdot$ \\
\hline RNM-1 & Cambric & $9 / 4 / 79$ & pump & no/no & $=$ & - & 532 & $\cdot$ & - & - & - & $=$ & $=$ & - & $7.10 \mathrm{e}+6$ & $3.11 \mathrm{e}+6$ & $<8.88 \mathrm{e}-3$ & $<1.81 \mathrm{e}-4$ \\
\hline RNM-1 & Cambric & $10 / 5 / 81$ & pump & no $/$ no & 5 & - & 228 & - & - & $\overline{-}$ & $\div$ & - & 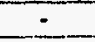 & $=$ & $3.04 \mathrm{e}+5$ & $1.33 e+5$ & $<8.88 \mathrm{e}-3$ & $<1.81 \mathrm{e}^{-4}$ \\
\hline RNM-1 & Cambric & $4 / 1 / 82$ & pump & no / no & - & - & 232 & - & - & - & - & - & - & - & $1.60 \mathrm{e}+5$ & $7.01 \mathrm{e}+4$ & $<8.88 \mathrm{e}-3$ & $<1.81 \mathrm{e}-4$ \\
\hline RNM-1 & Cambric & $4 / 10 / 85$ & - & no / .2 micron & - & - & $=$ & - & - & - & - & - & - & 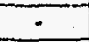 & $1.40 \mathrm{e}+4$ & $6.14 e+3$ & $<2.22 \mathrm{e}-3$ & $<4.52 \mathrm{e}-5$ \\
\hline RNM-1 & Cambric & $5 / 7 / 86$ & pump & no/no & - & - & - & - & - & $=$ & $\cdots$ & $=$ & $\div$ & - & $6.49 \mathrm{e}+3$ & $2.84 \mathrm{e}+3$ & - & - \\
\hline RNM-2S & Cambric & $11 / 29 / 78$ & pump & no/no & $=$ & - & 790 & $=$ & - & - & - & - & - & - & $4.00 \mathrm{e}+6$ & $1.75 \mathrm{e}+6$ & $<8.88 \mathrm{e}-3$ & $<1.81 \mathrm{e}-4$ \\
\hline RNM-2S & Cambric & $2 / 7 / 79$ & pump & no / no & $=$ & - & 579 & - & - & - & $=$ & - & - & - & $5.33 e+6$ & $2.34 \mathrm{e}+6$ & $<8.88 \mathrm{e}-3$ & $<1.81 \mathrm{e}-4$ \\
\hline RNM.2S & Cambric & $3 / 14 / 79$ & pump & no/no & $\div$ & - & 565 & - & $\therefore$ & $\because$ & $=$ & - & - & - & $5.84 e+6$ & $2.56 \mathrm{e}+6$ & $<8.88 \mathrm{e}-3$ & $<1.81 \mathrm{e}-4$ \\
\hline RNM-2S & Cambric & $8 / 29 / 79$ & pump & no / 1 micron & $\div$ & - & 389 & - & $=$ & $=$ & - & - & - & $=$ & $9.39 \mathrm{e}+6$ & $4.12 \mathrm{e}+6$ & $<8.88 \mathrm{e}^{-3}$ & $<1.81 \mathrm{e}-4$ \\
\hline RNM-2S & Cambric & $4 / 17 / 80$ & pump & no / 1 micron & - & - & 458 & - & - & - & - & - & - & $=$ & $1.35 \mathrm{e}+7$ & $5.94 e+6$ & $<8.88 \mathrm{e}-3$ & $<1.81 \mathrm{e}-4$ \\
\hline RNM-2S & Cambric & $7 / 23 / 80$ & pump & no / 1 micron & $\therefore$ & - & 282 & - & - & - & - & 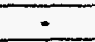 & - & $=$ & $1.39 \mathrm{e}+7$ & $6.07 \mathrm{e}+6$ & $<8.88 \mathrm{e}-3$ & $<1.81 \mathrm{e}-4$ \\
\hline RNM-2S & Cambric & $7 / 22 / 81$ & pump & no / 1 micron & $\div$ & - & 290 & - & $=$ & - & $\div$ & - & - & - & $1.34 \mathrm{e}+7$ & $5.86 \mathrm{e}+6$ & $<8.88 \mathrm{e}-3$ & $<1.81 \mathrm{e}-4$ \\
\hline RNM-2S & Cambric & $11 / 10 / 82$ & pump & no / I micron & - & - & 262 & - & 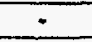 & - & 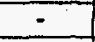 & - & - & - & $1.39 \mathrm{e}+7$ & $6.07 \mathrm{e}+6$ & $<8.88 \mathrm{e}-3$ & $<1.81 \mathrm{e}-4$ \\
\hline RNM-2S & Cambric & $4 / 19 / 84$ & pump & no / I micron & - & - & 300 & 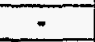 & - & - & 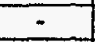 & $\because$ & - & - & $1.05 \mathrm{e}+7$ & $4.60 \mathrm{e}+6$ & $<8.88 \mathrm{e}-3$ & $<1.81 \mathrm{e}-4$ \\
\hline WW 5B & Cambric & $11 / 29 / 78$ & pump & no/no & 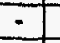 & - & 790 & - & - & - & 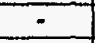 & - & - & - & - & - & $<4.44 e-3$ & $<8.01 e-5$ \\
\hline WW SC & \begin{tabular}{|l|} 
Cambric \\
\end{tabular} & $4 / 15 / 82$ & pump & no / 1 micron & - & - & 353 & - & $=$ & $E$ & - & - & - & $=$ & - & $=$ & $<4.44 e^{-3}$ & $<1.97 \mathrm{e}-4$ \\
\hline UE7ns & Bourbon & $6 / 24 / 83$ & pump & no /.45 micron & - & - & 322 & - & - & - & - & - & - & $=$ & $6.66 \mathrm{e}+3$ & $2.94 \mathrm{e}+3$ & $<4.44 e^{-3}$ & $<9.31 \mathrm{e}-5$ \\
\hline U20RPS\#1 & Cheshire & $9 / 8 / 83$ & pump & no / no & 8.4 & 3.1 & 256 & 0.163 & 73.5 & 0.187 & 13.4 & 2.43 & 1.87 & 4.5 & $4.02 \mathrm{e}+8$ & $2.25 e+8$ & $1.01 \mathrm{e}+0$ & $6.44 \mathrm{e}-2$ \\
\hline U20nPSH1 & Cheshire & $9 / 9 / 83$ & pump & no / no & 8.6 & 2.8 & 256 & 0.165 & 77.3 & 0.143 & 14 & 3.13 & 1.67 & 2.2 & $4.01 \mathrm{e}+8$ & $2.24 \mathrm{e}+8$ & $7.71 \mathrm{e}+0$ & $8.33 \mathrm{e}-2$ \\
\hline U20nPSH1 & Cheshire & $10 / 23 / 84$ & pump & no / no & - & $\div$ & 263 & - & - & - & $\therefore$ & - & 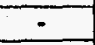 & - & $3.29 \mathrm{e}+8$ & $1.84 e+8$ & $1.07 \mathrm{e}+0$ & $6.82 \mathrm{e}-2$ \\
\hline U20nPS\#1 & Cheshire & $10 / 23 / 84$ & pump & no / no & - & - & 263 & - & - & $\because$ & - & - & - & - & $3.29 \mathrm{e}+8$ & $1.84 \mathrm{e}+8$ & $1.13 e+0$ & $7.23 \mathrm{e.2}$ \\
\hline $\begin{array}{l}\text { NOTE: } \\
\text { Detection } \\
\text { limit }=<\end{array}$ & & & & & & & & & & & & & & & & & & \\
\hline
\end{tabular}




\begin{tabular}{|c|c|c|c|c|c|c|c|c|c|c|c|c|c|c|}
\hline Well name & $\overline{\mathrm{NOK}}$ & $40 \mathrm{~K}$ & $60 \mathrm{Co}$ & $60 \mathrm{Co}$ & 106Ru & $106 \mathrm{Ru}$ & $125 \mathrm{Sb}$ & $125 \mathrm{Sb}$ & $137 \mathrm{Cs}$ & $137 \mathrm{Cs}$ & $155 \mathrm{Eu}$ & 155Eu & $235 U$ & $238 \mathrm{U}$ \\
\hline Unit & $(p C i / L)$ & $(p C i / L)$ & $(p \mathrm{Ci} / \mathrm{L})$ & $(p C i / L)$ & $(p \mathrm{C} i / L)$ & $(p \mathrm{Ci} / L)$ &.$(p C i / L)$ & $(p C \mathrm{C} / \mathrm{L})$ & $(p C i / L)$ & $(p C i / L)$ & $(p \mathrm{C} i / L)$ & $(p \mathrm{Ci} / \mathrm{L})$ & $(p C i / L)$ & $(p \mathrm{Ci} / \mathrm{L})$ \\
\hline Half-life (a) & & $1.25 e+9$ & & 5.271 & & 1.02 & & 2.758 & & 30.17 & & 4.73 & $7.40 e+8$ & $4.47 e+9$ \\
\hline Ref. date & collect. & $1 / 1 / 94$ & collect. & $1 / 1 / 94$ & collect. & $1 / 1 / 94$ & collect. & $1 / 1 / 94$ & collect. & $1 / 1 / 94$ & collect. & $1 / 1 / 94$ & collect. & collect. \\
\hline U3en & $1.07 e+2$ & $1.07 \mathrm{e}+2$ & $1.51 \mathrm{e}+0$ & $1.78 \mathrm{e}-1$ & $<4.88 \mathrm{e}+0$ & $87.79 e^{-5}$ & $4.22 \mathrm{e}+0$ & $7.09 \mathrm{e}-2$ & $1.60 \mathrm{e}+1$ & $1.10 \mathrm{e}+1$ & $<8.88 e-3$ & $<8.20 \mathrm{e}-4$ & $<3.77 \mathrm{e}-1$ & $1.33 e+2$ \\
\hline $\mathrm{U} 3 \mathrm{en}$ & $1.02 \mathrm{e}+2$ & $1.02 \mathrm{e}+2$ & $1.53 \mathrm{e}+0$ & $1.81 \mathrm{e}-1$ & $<5.33 e+0$ & $<8.50 \mathrm{e}-5$ & $5.11 \mathrm{e}+0$ & $8.59 \mathrm{e}-2$ & $1.64 \mathrm{e}+1$ & $1.13 e+1$ & $<8.88 \mathrm{e}-3$ & $<8.20 \mathrm{e}-4$ & $4.00 \mathrm{e}+0$ & $1.06 \mathrm{e}+2$ \\
\hline U3en & $1.03 e+2$ & $1.03 e+2$ & $1.47 \mathrm{e}+0$ & $1.73 \mathrm{e}-1$ & $<5.33 \mathrm{e}+0$ & $<8.50 \mathrm{e}-5$ & $5.11 \mathrm{e}+0$ & $8.59 \mathrm{e}-2$ & $1.84 \mathrm{e}+1$ & $1.27 \mathrm{e}+1$ & $<8.88 \mathrm{e}-3$ & $<8.20 \mathrm{e}-4$ & $4.22 \mathrm{e}+0$ & $1.25 \mathrm{e}+2$ \\
\hline U3cn & $6.30 \mathrm{e}+1$ & $6.30 \mathrm{e}+1$ & $<3.77 \mathrm{e}-1$ & $<4.45 \mathrm{e}-2$ & $<4.00 \mathrm{e}+0$ & $<6.37 \mathrm{e}-5$ & $3.33 \mathrm{e}+0$ & $5.60 \mathrm{e}-2$ & $1.33 e+1$ & $9.14 \mathrm{e}+0$ & $<8.88 \mathrm{e}-3$ & $<8.20 \mathrm{e}-4$ & $1.11 \mathrm{e}+0$ & $<4.08 \mathrm{e}+0$ \\
\hline U3en & $1.28 \mathrm{e}+2$ & $1.28 \mathrm{e}+2$ & $1.38 \mathrm{e}+0$ & $1.62 \mathrm{e}-1$ & $<3.33 \mathrm{e}+0$ & $<5.31 \mathrm{e}-5$ & $6.22 \mathrm{e}+0$ & $1.05 \mathrm{e}-1$ & $2.13 e+1$ & $1.47 \mathrm{e}+1$ & $<8.88 \mathrm{e}-3$ & $<8.20 \mathrm{e}-4$ & $4.22 \mathrm{e}+0$ & $1.29 \mathrm{e}+2$ \\
\hline U3en & $6.40 \mathrm{e}+1$ & $6.40 \mathrm{e}+1$ & $<4.00 \mathrm{e}-1$ & $<4.71 \mathrm{e}-2$ & $<4.00 \mathrm{e}+0$ & $<6.37 \mathrm{e}-5$ & $<1.35 \mathrm{e}+0$ & $<2.28 \mathrm{e}-2$ & $1.47 e+1$ & $1.01 \mathrm{e}+1$ & $<8.88 \mathrm{e}-3$ & $<8.20 \mathrm{e}-4$ & $<3.77 \mathrm{e}^{-1}$ & $<4.08 \mathrm{e}+0$ \\
\hline U3en & $7.20 \mathrm{e}+1$ & $7.20 \mathrm{e}+1$ & $<5.99 \mathrm{e}-1$ & $<7.07 \mathrm{e}-2$ & $<4.44 \mathrm{e}+0$ & $<7.08 \mathrm{e}-5$ & $3.11 \mathrm{e}+0$ & $5.23 e-2$ & $1.47 \mathrm{e}+1$ & $1.01 \mathrm{e}+1$ & $<8.88 \mathrm{e}-3$ & $<8.20 \mathrm{e}-4$ & $<3.77 e-1$ & $<4.08 \mathrm{e}+0$ \\
\hline $\mathrm{U} 3 \mathrm{en}$ & $7.91 \mathrm{e}+1$ & $7.91 \mathrm{e}+1$ & $3.33 e-1$ & $3.93 e-2$ & $8.66 \mathrm{e}+0$ & $1.38 \mathrm{e}-4$ & $4.66 \mathrm{e}+0$ & $7.84 \mathrm{e}-2$ & $1.49 \mathrm{e}+1$ & $1.02 e+1$ & $<8.88 \mathrm{e}-3$ & $<8.20 \mathrm{e}-4$ & $1.33 e+0$ & $3.27 \mathrm{e}+1$ \\
\hline U3cn & $31 \mathrm{e}+1$ & $6.31 \mathrm{e}+1$ & $6.66 \mathrm{e}-2$ & $7.86 \mathrm{e}-3$ & $<1.58 \mathrm{e}+0$ & $<2.51 \mathrm{e}-5$ & $1.33 e+0$ & $2.24 \mathrm{e}-2$ & $7.99 \mathrm{e}+0$ & $5.50 \mathrm{e}+0$ & $<8.88 \mathrm{e}-3$ & $<8.20 \mathrm{e}-4$ & $8.88 \mathrm{e}-1$ & $1.99 \mathrm{e}+1$ \\
\hline U3en & $31 \mathrm{e}+1$ & $7.31 \mathrm{e}+1$ & $2.22 \mathrm{e}-1$ & $2.62 e-2$ & $<2.22 \mathrm{e}-2$ & $<3.54 \mathrm{e}-7$ & $4.49 \mathrm{e}+0$ & $7.56 \mathrm{e}-2$ & $1.64 \mathrm{e}+1$ & $1.13 e+1$ & $<2.22 \mathrm{e}-2$ & $<2.05 e-3$ & $8.09 e-1$ & $1.72 \mathrm{e}+1$ \\
\hline U3cn-n5 & $55 \mathrm{e}+1$ & $3.55 \mathrm{e}+1$ & $<6.66 \mathrm{e}-3$ & $<7.86 \mathrm{e}-4$ & $<8.88 \mathrm{e}-2$ & $<1.42 \mathrm{e}-6$ & $<2.22 \mathrm{e}-2$ & $<3.73 e-4$ & $1.33 \mathrm{e}-2$ & $9.17 e-3$ & $4.44 e-1$ & $4.10 e-2$ & $2.22 \mathrm{e}-1$ & $2.91 \mathrm{e}+0$ \\
\hline U3en-n5 & $96 e+1$ & $2.96 \mathrm{e}+1$ & $<6.66 \mathrm{e}-3$ & $27.86 \mathrm{e}^{-4}$ & $<1.55 \mathrm{e}-1$ & $<2.48 \mathrm{e}-6$ & $<2.22 \mathrm{e}-2$ & $<3.73 e^{-4}$ & $6.66 \mathrm{e}-3$ & $4.58 \mathrm{e}-3$ & $4.44 \mathrm{e}-1$ & $4.10 \mathrm{e}-2$ & $1.78 \mathrm{e}-1$ & $2.42 e+0$ \\
\hline UE2ce & $30 \mathrm{e}+1$ & $9.30 \mathrm{e}+1$ & $<4.44 \mathrm{e}-3$ & $<5.56 \mathrm{e}-4$ & $4.44 \mathrm{e}-1$ & $9.65 \mathrm{e}-6$ & $<6.66 \mathrm{e}-2$ & $<1.26 \mathrm{e}-3$ & $<2.22 \mathrm{e}-2$ & $<1.54 \mathrm{e}-2$ & $2.66 \mathrm{e}-1$ & $2.63 e-2$ & $4.88 \mathrm{e}-1$ & $9.26 \mathrm{e}+0$ \\
\hline UE2ce & $5.22 e+1$ & $5.22 \mathrm{e}+1$ & $<4.44 e-3$ & $<5.56 \mathrm{e}-4$ & $1.11 \mathrm{e}+0$ & $2.41 e-5$ & $<4.44 \mathrm{e}-2$ & $<8.37 \mathrm{e}-4$ & $4.44 \mathrm{e}-2$ & $3.09 \mathrm{e}-2$ & $1.55 e-1$ & $1.53 e-2$ & $3.33-1$ & $8.79 \mathrm{e}+0$ \\
\hline UE2ce & $7.18 \mathrm{e}+1$ & $7.18 \mathrm{e}+1$ & $<4.44 \mathrm{e}-3$ & $<5.56 \mathrm{e}-4$ & $4.44 \mathrm{e}-1$ & $9.65 \mathrm{e}-6$ & $<4.44 \mathrm{e}-2$ & $<8.37 \mathrm{e}-4$ & $8.88 \mathrm{e}-2$ & $6.18 \mathrm{e}-2$ & $2.22 e-1$ & $2.19 \mathrm{e}-2$ & $3.33 e-1$ & $6.93 \mathrm{e}+0$ \\
\hline UE2ce & $.08 \mathrm{e}+1$ & $9.08 \mathrm{e}+1$ & $<4.44 \mathrm{e}-3$ & $<5.56 \mathrm{e}-4$ & $2.89 \mathrm{e}+0$ & $6.27 e-5$ & $<4.44 \mathrm{e}-2$ & $<8.37 e-4$ & $6.66 \mathrm{e}-2$ & $4.63 \mathrm{e}-2$ & $8.88 \mathrm{e}-2$ & $8.77 e-3$ & $6.22 \mathrm{e}-1$ & $4.73 e+0$ \\
\hline UE2ce & $5.52 \mathrm{e}+1$ & $5.52 \dot{e}+1$ & $6.88 \mathrm{e}-1$ & $8.62 \mathrm{e}-2$ & $1.33 \mathrm{e}-1$ & $2.89 e-6$ & $<2.66 \mathrm{e}-1$ & $<5.02 \mathrm{e}-3$ & $3.11 \mathrm{e}-1$ & $2.16 \mathrm{e}-1$ & $7.99 \mathrm{e}-1$ & $7.89 e-2$ & $2.44 \mathrm{e}-1$ & $5.06 \mathrm{e}+0$ \\
\hline UE2ce & $9.06 \mathrm{e}+1$ & $9.06 \mathrm{e}+1$ & $<4.44-3$ & $<5.56 \mathrm{e}-4$ & $1.33 \mathrm{e}-1$ & $2.89 \mathrm{e}-6$ & $<2.00 \mathrm{e}-1$ & $<3.77 \mathrm{e}-3$ & $3.33 e-2$ & $2.32 \mathrm{e}-2$ & $8.44 \mathrm{e}-1$ & $8.33 e-2$ & $4.22 \mathrm{e}-1$ & $9.21 \mathrm{e}+0$ \\
\hline UE2ce & 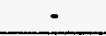 & - & - & - & - & - & - & - & - & - & 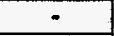 & - & - & - \\
\hline RNM-1 & $3.38 \mathrm{e}+1$ & $3.38 \mathrm{e}+1$ & $<4.44 \mathrm{e}-3$ & $<6.48 \mathrm{e}-4$ & $<5.99 \mathrm{e}-1$ & $<288 \mathrm{e}-5$ & $5.77 \mathrm{e}-1$ & $1.46 e-2$ & $1.88 \mathrm{e}+2$ & $1.34 \mathrm{e}+2$ & $<8.88 \mathrm{e}-3$ & $<1.04 \mathrm{e}-3$ & $7.10-1$ & $1.03 e+1$ \\
\hline RNM-1 & $2.51 \mathrm{e}+1$ & $2.51 \mathrm{e}+1$ & $<4.44 \mathrm{e}-3$ & $<6.48 \mathrm{e}-4$ & $<6.22 \mathrm{e}-1$ & $<2.98 \mathrm{e}-5$ & $4.44 \mathrm{e}-1$ & $1.12 \mathrm{e}-2$ & $8.30 \mathrm{e}+1$ & $5.93 e+1$ & $<8.88 \mathrm{e}-3$ & $<1.04 \mathrm{e}-3$ & $3.33 \mathrm{e}-1$ & $6.19 e+0$ \\
\hline RNM-1 & $2.94 \mathrm{e}+1$ & $2.94 \mathrm{e}+1$ & $<7.77 \mathrm{e}-3$ & $<1.13 \mathrm{e}-2$ & $<1.40 \mathrm{e}+0$ & $<6.71 \mathrm{e}-5$ & $<1.55 \mathrm{e}-1$ & $<3.93 \mathrm{e}-3$ & $8.24 \mathrm{e}+1$ & $5.88 \mathrm{e}+1$ & $<8.88 \mathrm{e}-3$ & $<1.04 \mathrm{e}-3$ & $4.00 \mathrm{e}-1$ & $8.48 \mathrm{e}+0$ \\
\hline RNM-1 & $2.53 e+1$ & $2.53 e+1$ & $<2.22 \mathrm{e}-3$ & $<3.24 \mathrm{e}-4$ & $<6.66 \mathrm{e}-2$ & $<3.20 \mathrm{e}-6$ & $4.44 \mathrm{e}-2$ & $1.12 \mathrm{e}-3$ & $5.47 \mathrm{e}+1$ & $3.91 \mathrm{e}+1$ & $<4.44 \mathrm{e}-2$ & $<5.20 \mathrm{e}-3$ & $1.78 \mathrm{e}-1$ & $2.58 \mathrm{e}+0$ \\
\hline RNM-1 & - & - & $=$ & - & - & - & - & - & - & - & - & - & - & - \\
\hline RNM-2S & $4.96 \mathrm{e}+1$ & $4.96 \mathrm{e}+1$ & $<4.44 \mathrm{e}-3$ & $<6.48 \mathrm{e}-4$ & $3.55 e-1$ & $1.70 e-5$ & $<4.44 e-2$ & $<1.12 \mathrm{e}-3$ & $8.88 \mathrm{e}-3$ & $6.34 \mathrm{e}-3$ & $<8.88 \mathrm{e}-3$ & $<1.04 e-3$ & $4.44 \mathrm{e}-1$ & $1.23 \mathrm{e}+1$ \\
\hline RNM-2S & $3.58 \mathrm{e}+1$ & $3.58 \mathrm{e}+1$ & $<4.44 \mathrm{e}-3$ & $<6.48 \mathrm{e}-4$ & $3.55 e-1$ & $1.70 e-5$ & $<4.44-2$ & $<1.12 \mathrm{e}-3$ & $<1.33 e-2$ & $<9.52 \mathrm{e}-3$ & $<8.88 \mathrm{e}-3$ & $<1.04 \mathrm{e}-3$ & $2.66 \mathrm{e}-1$ & $5.53 \mathrm{e}+0$ \\
\hline RNM-2S & $3.83 e+1$ & $3.83 \mathrm{e}+1$ & $<4.44 \mathrm{e}-3$ & $<6.48 \mathrm{e}-4$ & $3.33 \mathrm{e}-1$ & $1.60 \mathrm{e}-5$ & $<4.44 \mathrm{e}-2$ & $<1.12 \mathrm{e}-3$ & $<1.33 e-2$ & $<9.52 \mathrm{e}-3$ & $<8.88 \mathrm{e}-3$ & $<1.04 \mathrm{e}-3$ & $3.77 \mathrm{e}-1$ & $7.88 \mathrm{e}+0$ \\
\hline RNM-2S & $5.09 \mathrm{e}+1$ & $5.09 e+1$ & $<4.44 \mathrm{e}-3$ & $<6.48 \mathrm{e}-4$ & $6.88 \mathrm{e}-1$ & $3.30 \mathrm{e}-5$ & $2.22 \mathrm{e}-2$ & $5.61 \mathrm{e}-4$ & $2.66 \mathrm{e}-2$ & $1.90 \mathrm{e}-2$ & $<8.88 \mathrm{e}-3$ & $<1.04 \mathrm{e}-3$ & $2.00 \mathrm{e}-1$ & $4.22 \mathrm{e}+0$ \\
\hline RNM-2S & $6.35 e+1$ & $6.35 \mathrm{e}+1$ & $<4.44-3$ & $<6.48 \mathrm{e}-4$ & $4.00 \mathrm{e}-1$ & $1.92 \mathrm{e}-5$ & $<2.22 \mathrm{e}-2$ & $<5.61 \mathrm{e}-4$ & $5.33 e-2$ & $3.81 \mathrm{e}-2$ & $<8.88 \mathrm{e}-3$ & $<1.04 \mathrm{e}-3$ & $6.66 \mathrm{e}-1$ & $1.64 \mathrm{e}+1$ \\
\hline RNM-2S & $4.20 \mathrm{e}+1$ & $4.20 \mathrm{e}+1$ & $<4.44-3$ & $<6.48 \mathrm{e}-4$ & $4.88 \mathrm{e}-1$ & $2.34 \mathrm{e}-5$ & $<2.22 \mathrm{e}-2$ & $<5.61 e-4$ & $2.22 \mathrm{e}-2$ & $1.59 \mathrm{e}-2$ & $<8.88 \mathrm{e}-3$ & $<1.04 \mathrm{e}-3$ & $4.22 \mathrm{e}-1$ & $1.05 e+1$ \\
\hline RNM-2S & $3.47 \mathrm{e}+1$ & $3.47 \mathrm{e}+1$ & $<4.44-3$ & $<6.48 \mathrm{e}-4$ & $<4.44 \mathrm{e}-2$ & $<2.13 e-6$ & $<2.22 \mathrm{e}-2$ & $<5.61 \mathrm{e}-4$ & $6.22 \mathrm{e}-2$ & $4.44 \mathrm{e}-2$ & $<8.88 \mathrm{e}-3$ & $<1.04 \mathrm{e}-3$ & $2.89-1$ & $6.24 \mathrm{e}+0$ \\
\hline RNM-2S & $3.99 \mathrm{e}+1$ & $3.99 \mathrm{e}+1$ & $<4.44 e-3$ & $<6.48 \mathrm{e}-4$ & $<2.22 \mathrm{e}-2$ & $<1.07 \mathrm{e}-6$ & $<2.22 \mathrm{e}-2$ & $<5.61 \mathrm{e}-4$ & $3.33 e-2$ & $2.38 \mathrm{e}-2$ & $<8.88 \mathrm{e}-3$ & $<1.04 \mathrm{e}-3$ & $3.24 \mathrm{e}-1$ & $5.55 e+0$ \\
\hline RNM-2S & $4.05 e+1$ & $4.05 e+1$ & $<4.44 \mathrm{e}-3$ & $<6.48 \mathrm{e}-4$ & $<4.44 e-2$ & $<2.13 e-6$ & $<6.66 \mathrm{e}-2$ & $<1.68 \mathrm{e}-3$ & $<6.66 \mathrm{e}-3$ & $<4.76 \mathrm{e}-3$ & $<8.88 \mathrm{e}-3$ & $<1.04 \mathrm{e}-3$ & $4.00 \mathrm{e}-1$ & $5.42 e+0$ \\
\hline WW 5B & $4.27 \mathrm{e}+1$ & $4.27 \mathrm{e}+1$ & $<4.44 \mathrm{e}-3$ & $<6.10 \mathrm{e}-4$ & $<1.55 \mathrm{e}-1$ & $<5.47 \mathrm{e}-6$ & $<4.44 \mathrm{e}-2$ & $<1.00 \mathrm{e}-3$ & $<8.88 \mathrm{e}-3$ & $<6.28 \mathrm{e}-3$ & $<8.88 \mathrm{e}-3$ & $<9.73 e-4$ & $3.55 \mathrm{e}-1$ & $9.61 \mathrm{e}+0$ \\
\hline WW 5C & $3.91 \mathrm{e}+1$ & $3.91 \mathrm{e}+1$ & $<4.44-3$ & $<9.51 \mathrm{e}-4$ & $<2.22 \mathrm{e}-2$ & $<7.74 \mathrm{e}-6$ & $<2.22 \mathrm{e}-2$ & $<1.17 \mathrm{e}-3$ & $8.88 \mathrm{e}-2$ & $6.78 \mathrm{e}-2$ & $<8.88 \mathrm{e}-3$ & $<1.60 \mathrm{e}-3$ & $4.88 \mathrm{e}-1$ & $6.39 \mathrm{e}+0$ \\
\hline UE7ns & $2.44 \mathrm{e}+1$ & $2.44 \mathrm{e}+1$ & $4.44 \mathrm{e}-2$ & $6.57 \mathrm{e}-3$ & $<1.11 \mathrm{e}-1$ & $<5.74 e-6$ & $<2.22 \mathrm{e}-2$ & $<5.77 \mathrm{e}-4$ & $2.00 \mathrm{e}-1$ & $1.43 \mathrm{e}-1$ & $<8.88 \mathrm{e}-3$ & $<1.06 \mathrm{e}-3$ & $1.55 \mathrm{e}-2$ & $5.99 \mathrm{e}-1$ \\
\hline U20nPS\#1 & $2.67 e+0$ & $2.67 \mathrm{e}+0$ & $2.04 \mathrm{e}+0$ & $5.24 \mathrm{e}-1$ & $7.24 \mathrm{e}+1$ & $6.45 e-2$ & $7.85 e+2$ & $5.86 \mathrm{e}+1$ & $2.68 \mathrm{e}+3$ & $2.11 \mathrm{e}+3$ & $1.35 \mathrm{e}+1$ & $2.97 \mathrm{e}+0$ & 5 & - \\
\hline U20nPS\#1 & $5.24 e+0$ & $5.24 \mathrm{e}+0$ & $2.24 \mathrm{e}+0$ & $5.76 \mathrm{e}-1$ & $8.57 \mathrm{e}+1$ & $7.63 e-2$ & $9.34 e+2$ & $6.97 \mathrm{e}+1$ & $2.86 \mathrm{e}+3$ & $2.25 \mathrm{e}+3$ & $2.01 \mathrm{e}+1$ & $4.42 \mathrm{e}+0$ & - & - \\
\hline U20nPS\#1 & $3.58 \mathrm{e}+0$ & $3.58 \mathrm{e}+0$ & $1.26 \mathrm{e}+0$ & $3.24 \mathrm{e}-1$ & $6.60 \mathrm{e}+1$ & $5.88 \mathrm{e}-2$ & $5.26 \mathrm{e}+3$ & $7.52 \mathrm{e}+1$ & $3.86 \mathrm{e}+3$ & $3.05 e+3$ & $1.27 \mathrm{e}+1$ & $2.80 \mathrm{e}+0$ & - & $=$ \\
\hline U20nPS\#1 & $4.15 e+0$ & $4.15 e+0$ & $1.15 e+0$ & $2.96 e-1$ & $6.08 \mathrm{e}+0$ & $5.41 \mathrm{e}-3$ & $1.09 \mathrm{e}+3$ & $8.11 \mathrm{e}+1$ & $3.69 \mathrm{e}+3$ & $2.91 \mathrm{e}+3$ & $1.14 e+1$ & $2.49 \mathrm{e}+0$ & $=$ & - \\
\hline $\begin{array}{l}\text { etection } \\
\text { nit }=<<\end{array}$ & & & & & & & & & & & & & & \\
\hline
\end{tabular}




\title{
III. GROUNDWATER PROTECTION
}

\section{TRANSPORT OF GASEOUS FISSION PRODUCTS ADJACENT TO AN UNDERGROUND NUCLEAR TEST CAVITY}

\author{
David K. Smith, Richard J. Nagle and Jacqueline M. Kenneally \\ INTRODUCTION
}

Radiochemical weapons diagnostics, containment strategies for underground nuclear testing as well as conceptual models of the subsurface transport of radionuclides rely on knowledge of the emplacement and distribution of radionuclides generated during an underground nuclear explosion. In 1994, scientists of the combined LLNL and LANL Joint Test Organization at the Nevada Test Site targeted two previously fired events for drilling to exercise the capability to re-enter an expended test, collect samples for radiochemical diagnostics and provide characterization of the near-field environment. The 1989 INGOT event was the first of two underground nuclear tests drilled back in 1994. While the event was previously drilled back in 1989 for radiochemical diagnostics, it was identified for re-drilling based on 1) its relatively young age, 2) extreme radiochemical fractionation noted during initial diagnostics, and 3) the strategic significance of the device tested. Table V summarizes information as it relates to the INGOT event as fielded in 1989. Of note, the INGOT event was fired above the static water level.

TABLE V

Hole

Drill Date

Drill Depth

Hole Diameter

Depth of Burial (DOB)

INGOT

Fire Date

Announced Yield

Time to Cavity Collapse

Depth to Static Water Level (SWL)

Geologic Formation at DOB

\author{
U2gg \\ $11 / 25 / 87$ \\ $555 \mathrm{~m}$ \\ $2.44 \mathrm{~m}$ \\ $500 \mathrm{~m}$ (vertical) \\ March 9, 1989 \\ $20-150 \mathrm{Kt}$ \\ $\mathrm{H}+45$ minutes \\ $565 \mathrm{~m}$ (vertical) \\ Timber Mountain Tuff
}

\section{DRILLING OPERATIONS}

The INGOT drill-back consisted of two separate holes. The first was slant drilled $18^{\circ}$ off-vertical back into the INGOT explosion cavity; six debris samples were returned for radiochemical diagnostics of device performance. Samples collected from the first hole will not be discussed further. The second hole was drilled to provide environmental characterization. The 
drilling rig was moved and the bit angled at $18^{\circ}$ to pass within $10 \mathrm{~m}$ of the edge of the cavity at the level of the working point $(500 \mathrm{~m})$ and bottom below the cavity at a total depth beneath the static water level. The $25 \mathrm{~cm}$ diameter hole was drilled to a total slant depth of $726.5 \mathrm{~m}$. Because the bore was angled, only slant - not true vertical - drilling depths are referenced. A conceptual illustration of this second hole is provided in Figure 3. The hole was then logged using a high sensitivity spectral gamma ray tool (Gadeken et al., 1988); this tool uses a large $(5 \mathrm{~cm} \times 30 \mathrm{~cm}) \mathrm{NaI}(\mathrm{Tl})$ detector coupled to a low noise photomultiplier and digital pulse height analyzer to transmit 256 channel spectra over the ranges $0-350 \mathrm{KeV}$ and $0-3000 \mathrm{KeV}$. The gamma log encountered one discrete zone of gamma activity between 520 and $540 \mathrm{~m}$ slant depth. The gamma log is presented in Figure 4. The only radionuclide identified in the spectral data was ${ }^{137} \mathrm{Cs}$. Based on the field logs, a sidewall tool was used to collect solid samples from this interval. Three samples were returned - two from a slant depth of $527 \mathrm{~m}$ and one from a slant depth of 537 m. After all logging runs were completed, $14 \mathrm{~cm}$ diameter stainless steel casing was run in the hole. The bottom $46 \mathrm{~m}$ of the casing was slotted below the depth of the pre-shot static water level to allow for subsequent groundwater sampling.

\section{ANALYTICAL RESULTS}

Solid samples were returned to the Isotope Sciences Division at LLNL for analysis. Gamma emitting radionuclides and ${ }^{90} \mathrm{Sr}$ were determined by $\gamma$ and $\beta$ counting respectively. Scanning electron microscopy (SEM) with energy dispersive analysis was used to characterize texture and determine major element chemistry. Minerals were identified by X-ray diffraction (XRD). The whole-rock samples were examined without washing or mineral-matrix separation except for the ${ }^{90} \mathrm{Sr}$ analyses where the samples were digested prior to separation of $\mathrm{Sr}$ and $\beta$ counting of the $90 \mathrm{Y}$ daughter.

\section{Radiochemistry}

The gamma and beta analyses indicate the variable presence of $40 \mathrm{~K}, 228 \mathrm{Th}$, ${ }^{137} \mathrm{Cs}$ and ${ }^{90} \mathrm{Sr}$ in each of the three samples. The data are summarized in Table VI. 


\section{INGOT (U2gg) DRILL-BACK}

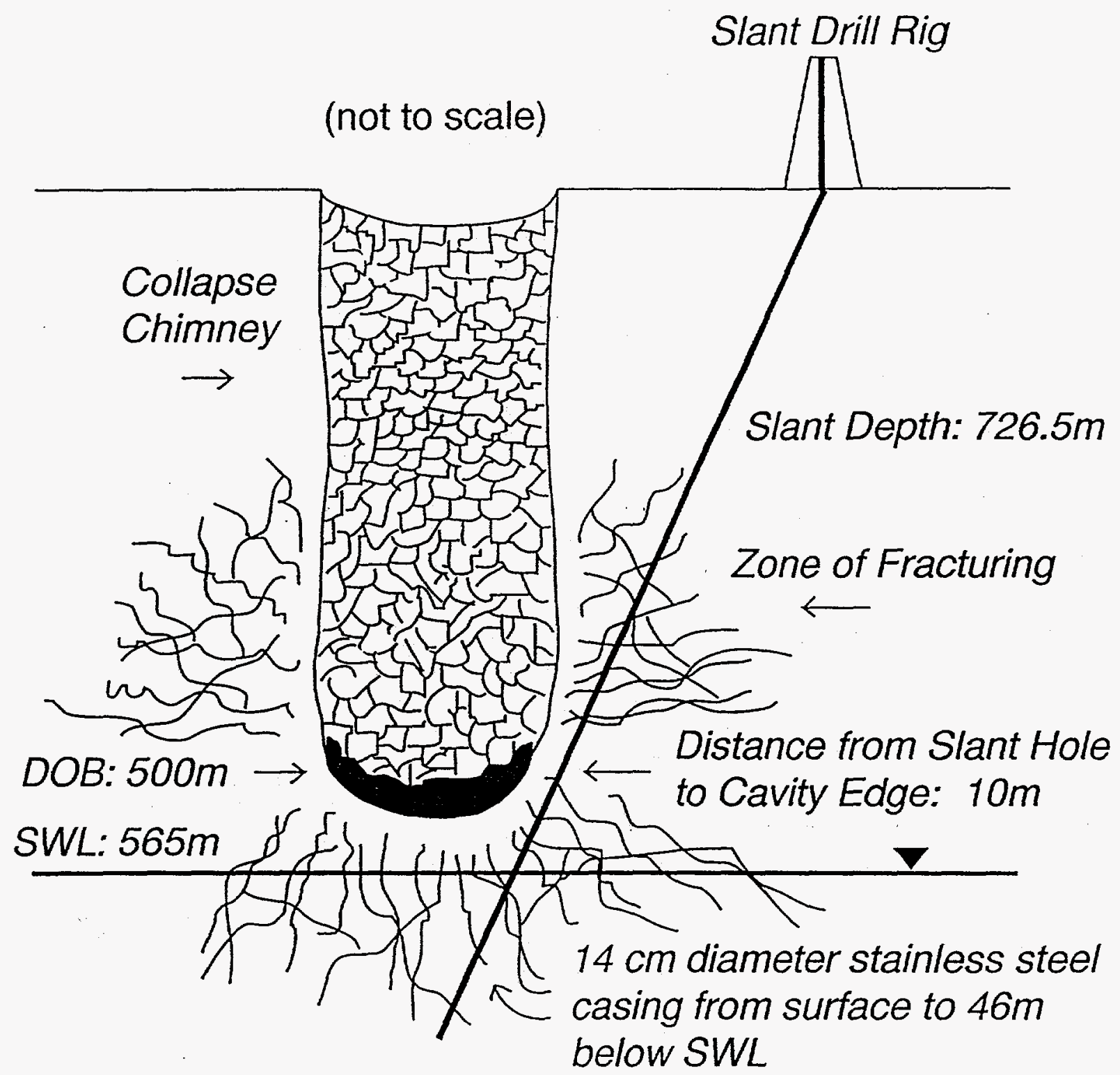

Figure 3: Conceptual illustration of INGOT (U2gg) drilling exercise adjacent to the edge of an underground nuclear test cavity. All drilling depths slant. $\mathrm{DOB}=$ Device depth of burial; $S W L=$ Static water level. Nuclear explosive melt debris is represented in black with the overlying collapse chimney extending to the surface. Zone of fracturing occurs within three cavity radii of the DOB. 


\section{Spectral Gamma Log}

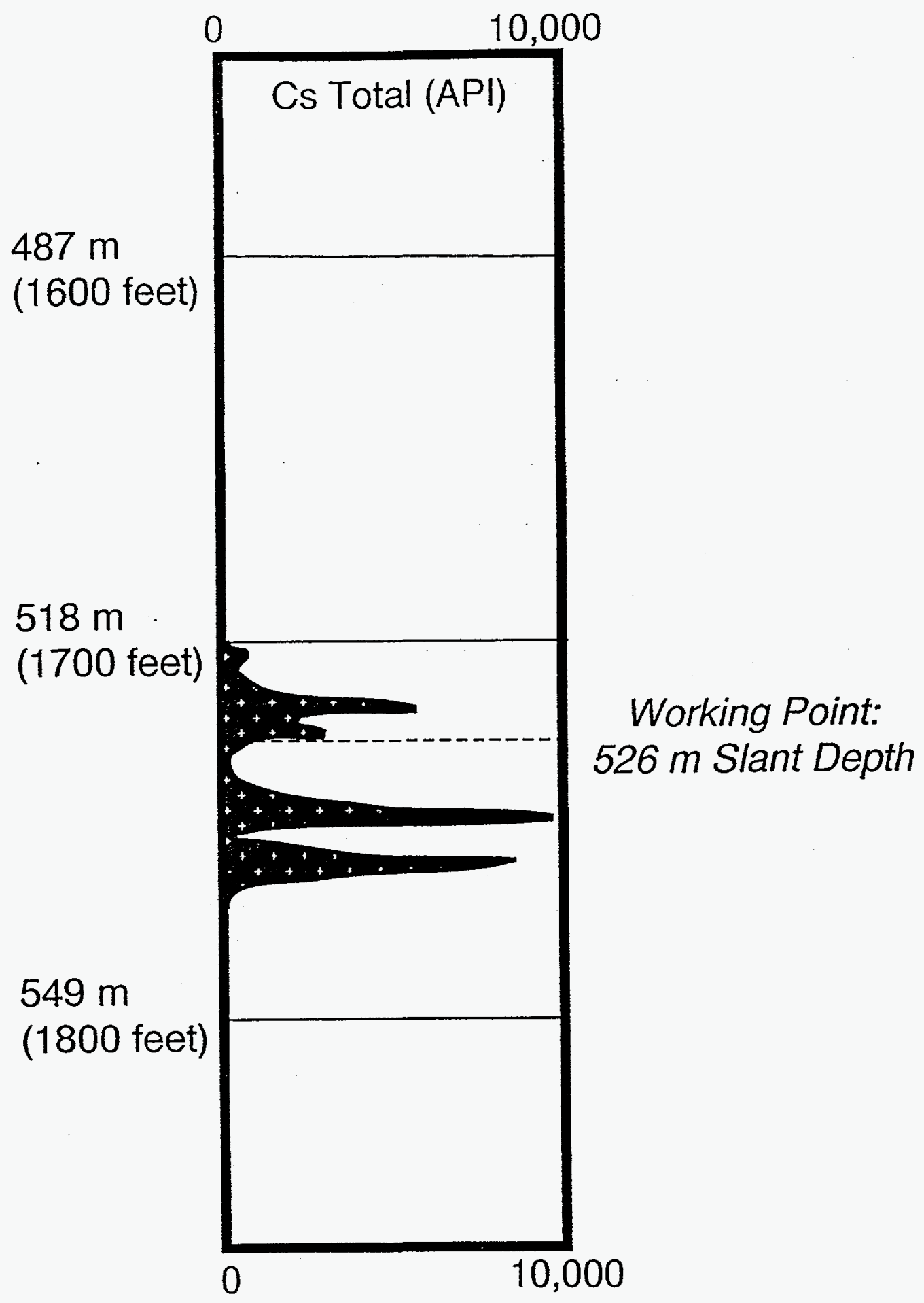

Figure 4: High sensitivity gamma log of the INGOT (U2gg) drill hole shown in Figure $3 .{ }^{137} \mathrm{Cs}$ activity confined to the interval between 500 and $550 \mathrm{~m}$. All drilling depths slant. 
TABLE VI

\section{SAMPLE C-1 $\quad$ SAMPLE C-3 $\quad$ SAMPLE C-8}

$\begin{array}{cccc}\text { SLANT DEPTH } & 537 \mathrm{~m}(1760 \text { feet }) & 527 \mathrm{~m}(1730 \text { feet }) & 527 \mathrm{~m}(1730 \text { feet }) \\ { }^{40} \mathrm{~K}^{\mathrm{a}} & 7.74 \times 10^{1} \pm 20.0 \% & 8.49 \times 10^{1} \pm 26.6 \% & 1.00 \times 10^{2} \pm 35.4 \% \\ { }^{228} \mathrm{Th}^{\mathrm{a}} & 2.33 \times 10^{1} \pm 29.3 \% & 4.69 \times 10^{1} \pm 31.2 \% & \\ { }^{137} \mathrm{Cs}^{\mathrm{a}} & 2.08 \times 10^{0} \pm 41.2 \% & 1.59 \times 10^{2} \pm 2.5 \% & 7.25 \times 10^{3} \pm 1.0 \% \\ { }^{90} \mathrm{Sr}^{\mathrm{a}} & 1.19 \times 10^{-1} \pm 8.4 \% & 1.78 \times 10^{-2} \pm 13.1 \% & 1.29 \times 10^{1} \pm 0.9 \% \\ { }^{137} \mathrm{Cs} /{ }^{90} \mathrm{Sr} & 1.75 \times 10^{1} & 8.93 \times 10^{3} & 5.62 \times 10^{2} \\ { }^{137} \mathrm{Cs} /{ }^{40} \mathrm{~K} & 2.69 \times 10^{-2} & 1.87 \times 10^{0} & 7.18 \times 10^{1}\end{array}$

a) all DPM / gram, corrected to zero (firing) time; counting errors in percent

${ }^{40} \mathrm{~K}$ and ${ }^{228} \mathrm{Th}$ are naturally present in silicic volcanic rocks and Nevada Test Site drilling muds (Esser, 1994). Because the sample matrix consisted predominantly of tuffs adjacent to the INGOT working point, the presence of ${ }^{40} \mathrm{~K}$ and ${ }^{228} \mathrm{Th}$ is not surprising. ${ }^{137} \mathrm{Cs}$ and ${ }^{90} \mathrm{Sr}$ are fission products created from the burn-up of primary nuclear fuels. These two radionuclides are unique because they are each produced in part from the decay of a gaseous parent. ${ }^{137} \mathrm{Cs}$ is produced from the decay of ${ }^{137} \mathrm{Xe}$ with a 229.2 second half-life; ${ }^{90} \mathrm{Sr}$ is produced from the decay of $90 \mathrm{Kr}$ with a 32.3 second half-life. The decay series for each of these species is provided in Table VII.

TABLE VII

\section{PRECURSORS $\quad \mathrm{t} 1 / 2$}

${ }^{137} \mathrm{Cs}$

$\begin{array}{llll} & { }^{137} \mathrm{Sb} & 0.284 \text { seconds } & 0.0025 \% \\ & { }^{137} \mathrm{Te} & 2.5 \text { seconds } & 0.4377 \% \\ & { }^{137} \mathrm{I} & 24.5 \text { seconds } & 2.56 \% \\ & { }^{137} \mathrm{Xe} & 229.2 \text { seconds } & 5.92 \% \\ & { }^{137} \mathrm{Cs} & 30.1 \text { years } & 6.09 \% \\ & & & \\ & & & \\ & & 0.55 \text { seconds } & 0.025 \% \\ & 90 \mathrm{Se} & 1.9 \text { seconds } & 0.56 \% \\ & 90 \mathrm{Br} & 32.3 \text { seconds } & 4.46 \% \\ & 90 \mathrm{Kr} & 258 \text { seconds } & 1.17 \% \\ & 90 \mathrm{Rb}{ }^{4-} & 156 \text { seconds } & 4.25 \%(90 \mathrm{Rb}: 5.42 \%) \\ & 90 \mathrm{Rb}{ }^{1-} & 29.1 \text { years } & 5.46 \% \\ & 90 \mathrm{Sr} & \end{array}$

${ }^{90} \mathrm{Sr}$

$\begin{array}{llll} & { }^{137} \mathrm{Sb} & 0.284 \text { seconds } & 0.0025 \% \\ & { }^{137} \mathrm{Te} & 2.5 \text { seconds } & 0.4377 \% \\ & { }^{137} \mathrm{I} & 24.5 \text { seconds } & 2.56 \% \\ & { }^{137} \mathrm{Xe} & 229.2 \text { seconds } & 5.92 \% \\ & { }^{137} \mathrm{Cs} & 30.1 \text { years } & 6.09 \% \\ & & & \\ & & & \\ & & 0.55 \text { seconds } & 0.025 \% \\ & 90 \mathrm{Se} & 1.9 \text { seconds } & 0.56 \% \\ & 90 \mathrm{Br} & 32.3 \text { seconds } & 4.46 \% \\ & 90 \mathrm{Kr} & 258 \text { seconds } & 1.17 \% \\ & 90 \mathrm{Rb}{ }^{4-} & 156 \text { seconds } & 4.25 \%(90 \mathrm{Rb}: 5.42 \%) \\ & 90 \mathrm{Rb}{ }^{1-} & 29.1 \text { years } & 5.46 \% \\ & 90 \mathrm{Sr} & \end{array}$

a) SNM: Special nuclear materials as actinide nuclear weapon fuels

CUMULATIVE SNM ${ }^{\mathrm{a}}$ FISSION CHAIN YIELD 
The fission product data are notable for several reasons. First, ${ }^{137} \mathrm{Cs}$ and ${ }^{90} \mathrm{Sr}$ are the only fission products detected in a discrete zone of radioactivity ten meters away from the edge of the INGOT cavity. No refractory radionuclides (i.e, ${ }^{95} \mathrm{Zr},{ }^{144} \mathrm{Ce},{ }^{155} \mathrm{Eu}$ ) were detected. Second, there is an appreciable enrichment in observed ${ }^{137} \mathrm{Cs}$ relative to observed ${ }^{90} \mathrm{Sr}$ compared to a ratio of the same radionuclides calculated from a radionuclide inventory for this event (Goishi et al., 1994). Due to the nature of the inventory data, only relative comparisons are provided. Third, the distribution of radionuclides is extremely heterogeneous; ${ }^{137} \mathrm{Cs}$ and ${ }^{90} \mathrm{Sr}$ activities and ${ }^{137} \mathrm{Cs} /{ }^{90} \mathrm{Sr}$ ratios vary over a maximum of three orders of magnitude.

Prompt gas samples collected over a five day period following the INGOT event indicated enrichments in $\mathrm{Kr} / \mathrm{Ar}$ ratios (10.8\%) and $\mathrm{Xe} / \mathrm{Ar}$ ratios (16.5\%) relative to ratios for the composition of normal air. Consistently elevated heavy rare gas concentrations attest to the ability of these gases to be transported pervasively and uniformly throughout the post-event explosion cavity complex.

\section{Scanning Electron Microscopy}

In bulk specimen, the whole-rock returns resemble the unwelded ash fall comprising the Timber Mountain Tuff in the vicinity of the working point. However, under the SEM, instead of a partially devitrified ash-rich matrix typical of such rocks, these samples display a partially fused matrix characterized by abundant vesicles and conchoidal fractures (Figure 5). These features are consistent with recent melting at the time of the explosion. The crystalline, devitrified textures typical of undisturbed tuffs are largely obliterated. The Timber Mountain Tuff melts at temperatures below $1000^{\circ} \mathrm{C}$ in part due to the high water contents of this rock (between 10 and 20 weight\%). Partial melting was also noted along fracture surfaces produced during the 1957 RAINIER event (Wadman and Richards, 1961).

\section{$X$-ray Diffraction}

Solid samples were also analyzed by XRD to identify the mineral constituents in the radioactive samples. The identification of any high temperature and/or high pressure phases together with discrepancies in the pre- and postevent XRD spectra would be indicative of significant bulk mineralogical changes associated with the underground detonation. Neither high P-T phases or substantive shifts from the pre-detonation lithology were observed. This is consistent with findings for the 1966 PILEDRIVER event (Borg, 1974). Cristobalite, quartz and $\mathrm{Na}-\mathrm{Ca}$ feldspars are identified in the post-detonation spectra (Figure 6) and are not unusual. Montmorillonite in excess of 30 weight\% is identified in the pre-shot sample at the depth of the INGOT working point $(500 \mathrm{~m})$; the corresponding post-shot sample from the same 


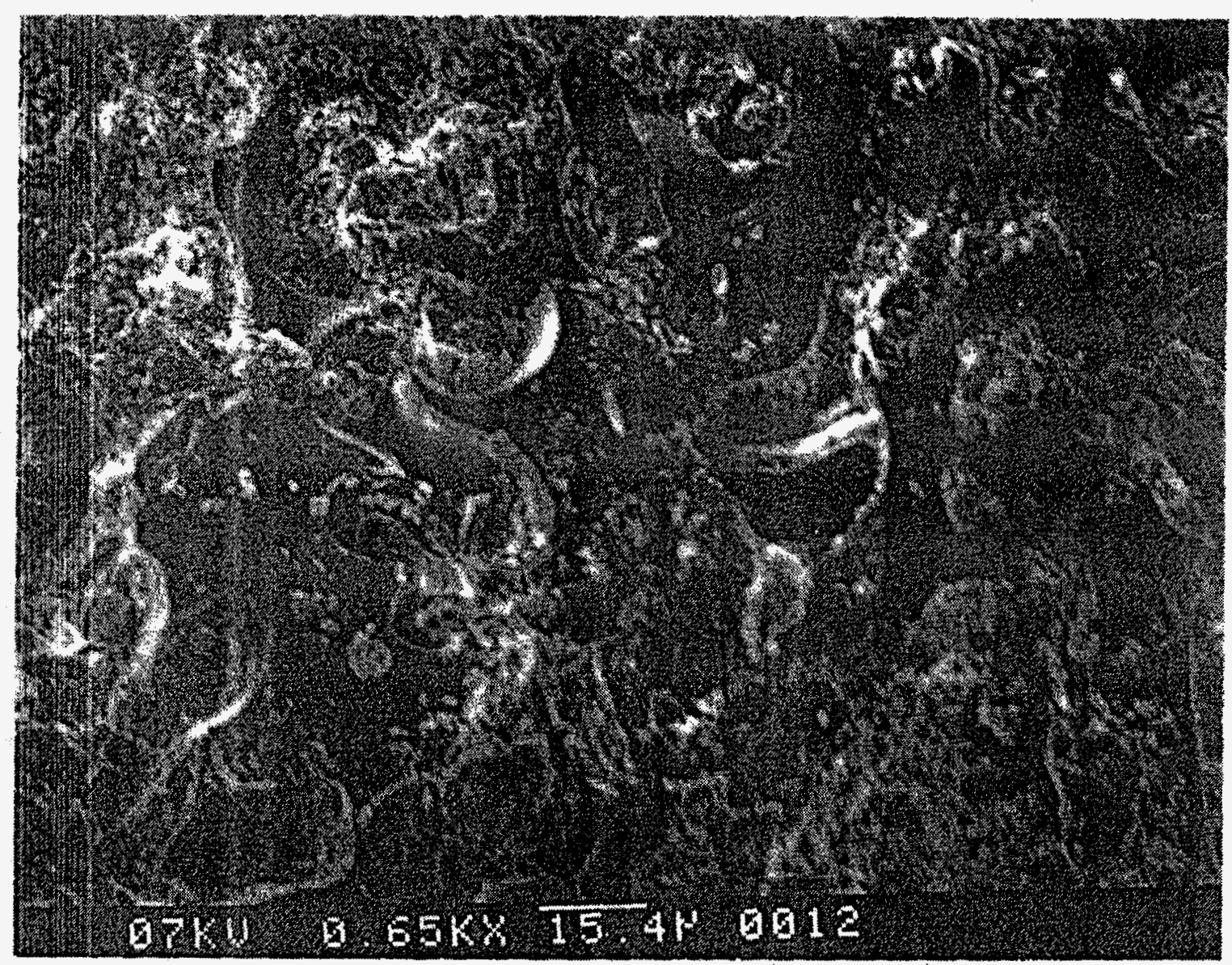

Figure 5: Scanning electron micrograph of partially fused vesicular glass returned from radioactive interval identified by the gamma log. $650 \mathrm{x}$ magnification. Temperatures associated with the explosion were high enough to melt the formation in excess of 10 meters away from the edge of the INGOT cavity. 


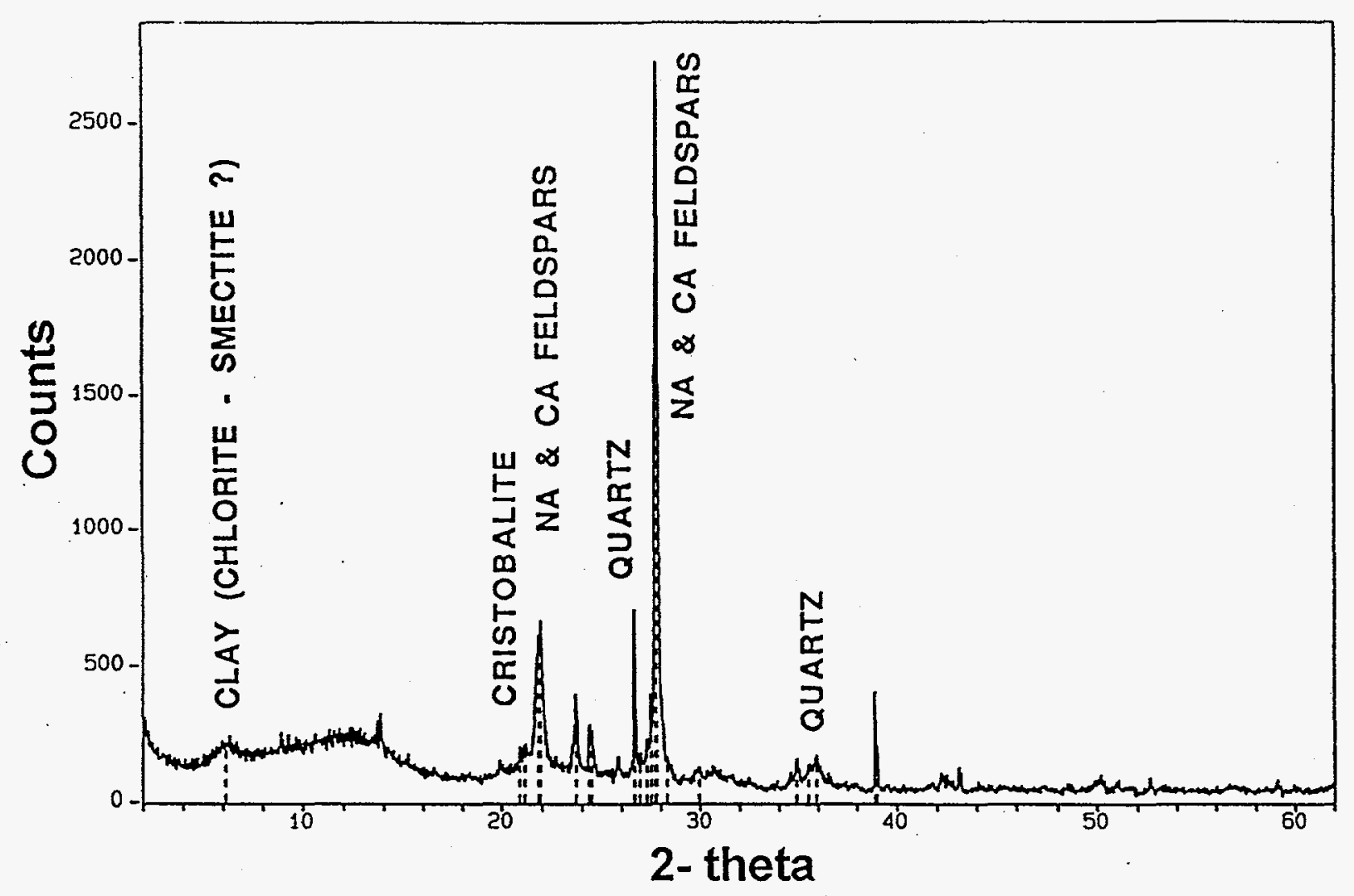

Figure 6: Post event $X$-ray pattern ( $2 \Theta$ verses intensity) with labeled peaks for a solid sample returned from a slant depth of $527 \mathrm{~m}$ adjacent to the edge of the INGOT cavity. Crystalline phases are not unexpected for the Timber Mountain Tuff. 
interval displays a weak $14.38 \AA$ A reflection for clay (montmorillonite or interlayered chlorite-smectite) indicating its presence as a minor constituent (Figure 6).

\section{ROLE OF FRACTURES}

The INGOT drilling exercise afforded a unique opportunity to investigate the distribution of radionuclides near the edge of a nuclear test cavity. Drilling outside of the cavity in the vadose zone insured that any observed radionuclide signatures would be the result of transport associated with the explosion and not transport by groundwater during or after the event. The distribution of radionuclides within three cavity radii of the INGOT working point may be controlled in part by fractures associated with the explosion. Reactivated pre-existing and shot-induced fissure surfaces are likely conduits for the extremely confined zone of radioactivity noted in the gamma log. OTA (1989) notes the inability of a extremely weak rock such as a water saturated clay to support the extreme stresses associated with an underground nuclear explosion without failure. Lithologic contacts between "strong" rock (welded tuff) and "weaker" rock (ash-fall tuff) are also prone to disruption along bedding surfaces (G. Pawloski, LLNL, 1995, pers. comm.).

Geologic and geophysical logs run in the INGOT (U2gg) emplacement hole prior to the explosion provide some indication of rock strength and formation integrity (Newmark and Schmidt, 1988). Density and caliper logs are used to distinguish zones potentially susceptible to mechanical failure. Excursions in these logs were observed within 10 to $20 \mathrm{~m}$ of the working point (Figure 7). At the working point horizon, the lithologic log indicates contact between nonwelded and welded and fractured members of the Timber Mountain Tuff. At the same interval, there is a significant increase in water content (to 23.5 weight\% $\mathrm{H}_{2} \mathrm{O}$ ). Changes in clay content with depth, determined by XRD, are shown in Figure 8 . Notably montmorillonite is identified throughout the interval from 491 to $512 \mathrm{~m}$ vertical depth which includes the working point at $500 \mathrm{~m}$. At a depth of $497 \mathrm{~m}$, montmorillonite content peaks at 30 weight\% of the rock mode. Changes in the mineralogy and mechanical anisotropy of the formation partially controls the distribution of fractures created by the explosion.

\section{DISCUSSION}

A conceptual model follows. At zero time the pressurized cavity gas is mostly steam. Calculations reported by Knox et al. (1967) for a theoretical cratering experiment in a silicate rock containing 10 weight $\% \mathrm{H}_{2} \mathrm{O}$ predict that the cavity environment will consist of melted $\mathrm{SiO}_{2}$ dispersed in superheated steam in a ratio of $1: 200\left(\mathrm{SiO}_{2}: \mathrm{H}_{2} \mathrm{O}\right)$ with a cavity pressure of 10 to 20 bars and a temperature of $2000^{\circ} \mathrm{C}$. The shock wave generated by the explosion forms a cavity and induces radial fracturing out to a distance of two cavity 


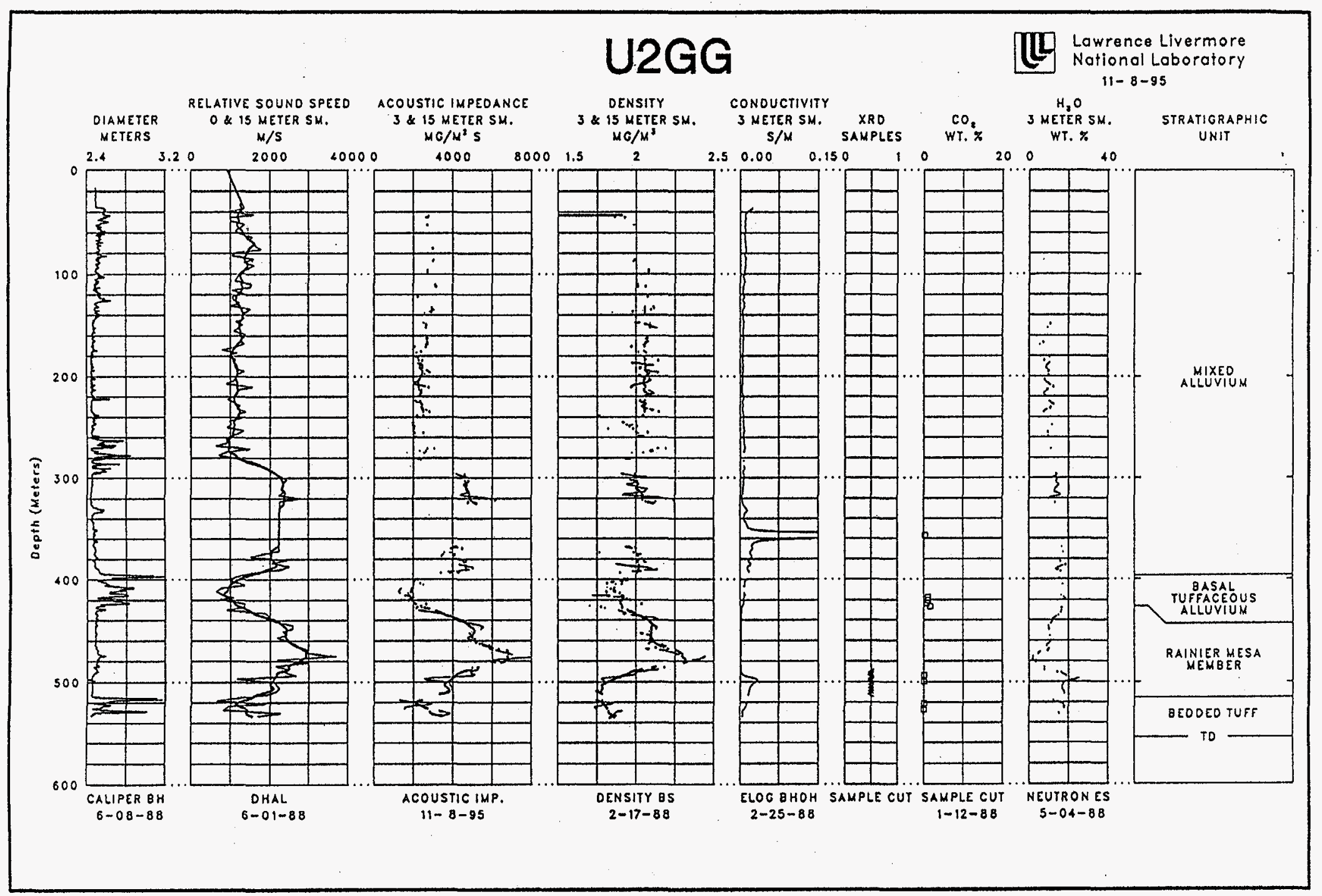

Figure 7: Pre-event geophysical and geologic logging suite for U2gg adapted from Newmark and Schmidt (1988). The INGOT working (firing) point is $500 \mathrm{~m}$ vertical depth. 


\section{INGOT (U2gg) LITHOLOGIC LOG}

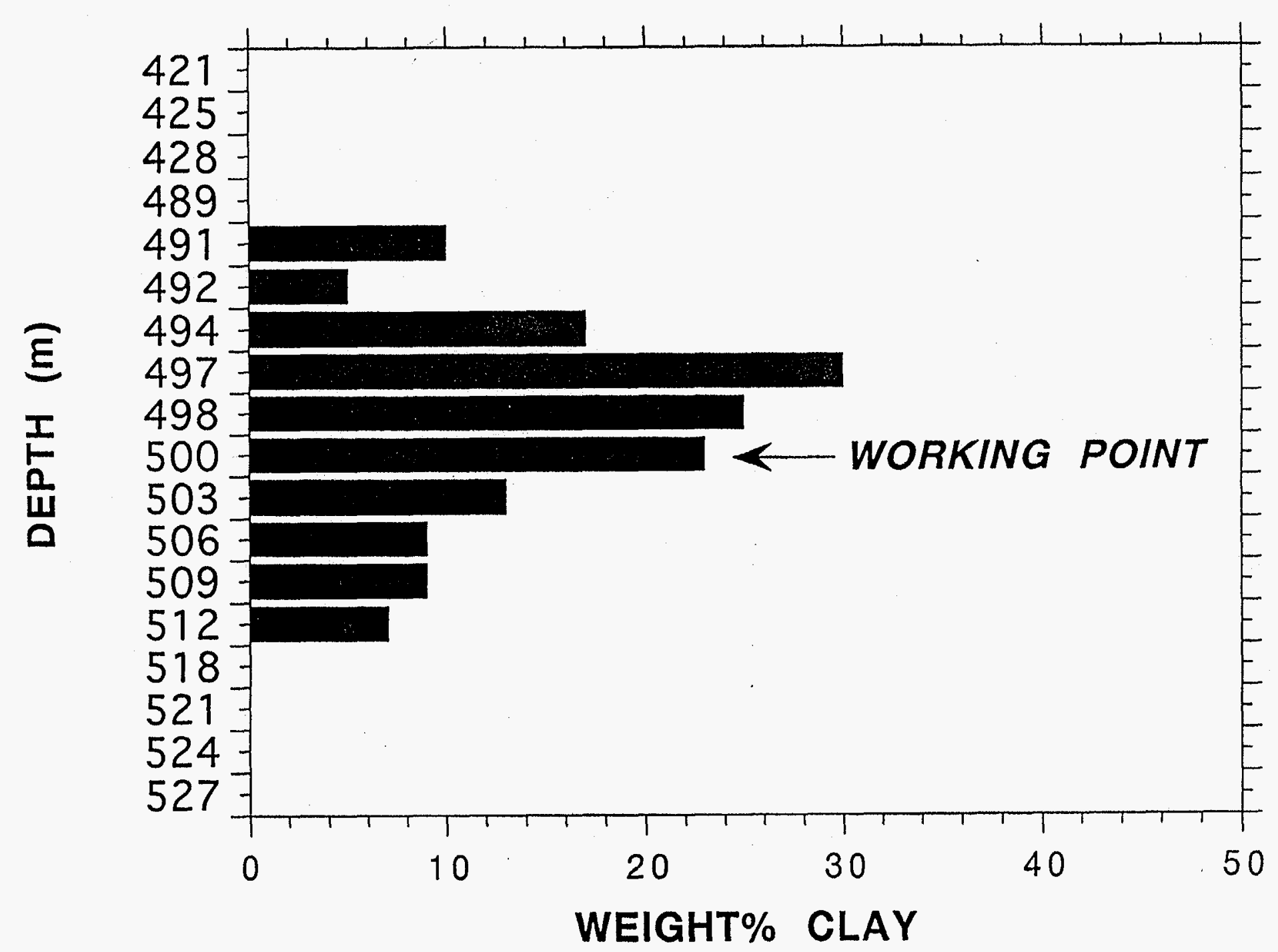

Figure 8: Pre-event lithologic log. The presence of clay relative to depth proximal to the INGOT working point. Note near the depth of burial montmorillonite (a hydrated clay) is measured up to 30 weight\%. 
radii away from the working point and reactivates, extends and interconnects existing fractures out to three cavity radii (OTA, 1989). Rock strength is inversely correlated to water content. The occurrence of hydrated ( $>20$ weight $\% \mathrm{H}_{2} \mathrm{O}$ ) smectites at the depth of burial results in a discrete interval of lower rock strength. A fracture begins to propagate along this horizon. Mechanical stresses from the expansion of steam rapidly promotes fracture growth (Butkovich and Lewis, 1973). The water contents in the clay-rich horizons are high enough ( 23.5 weight\%) to promote melting of the volcanic ash that is exposed along the fracture surfaces. Temperatures in excess of $1000^{\circ} \mathrm{C}$ are possible ten meters or more away from ground zero (Butkovich, 1974).

Of significance is the distribution of the two volatile gaseous precursors to ${ }^{90} \mathrm{Rb}\left({ }^{90} \mathrm{Sr}\right)$ and ${ }^{137} \mathrm{Cs}$. The 32.3 second and 229.2 second half lives of the parent isotopes ${ }^{90} \mathrm{Kr}$ and ${ }^{137} \mathrm{Xe}$ allow these radionuclides to be transported as gases before decay to their longer-lived daughters. Rather than a pressuretemperature pulse which 'promptly injects' a plasma which condenses volatile and refractory radionuclides, the results from INGOT suggest that some nuclear test cavities many exhibit an irregular perimeter defined by volatile radionuclides transported in late-time along fractures created by the explosion. INGOT ${ }^{137} \mathrm{Cs} /{ }^{90} \mathrm{Sr}$ atom ratios are severely fractionated relative to ratios calculated from the INGOT radionuclide inventory (Goishi et al., 1994). These results suggest the late-time gaseous transport of $90 \mathrm{Kr}$ and ${ }^{137} \mathrm{Xe}$ from the cavity through fractures is responsible for the dispersion and subsequent deposition of ${ }^{137} \mathrm{Cs}$ and ${ }^{90} \mathrm{Rb}\left({ }^{90} \mathrm{Sr}\right)$. The extreme ratio of ${ }^{137} \mathrm{Cs} /{ }^{90} \mathrm{Sr}$ for the INGOT samples relative to the ratio calculated from the event-specific radionuclide inventory likely reflects the preferential decay of ${ }^{90} \mathrm{Kr}\left(t_{1 / 2}: 32.3\right.$ seconds) relative to ${ }^{137} \mathrm{Xe}$ ( $t_{1 / 2}: 229.2$ seconds) during the time required for the gaseous phase to travel through the fracture prior to deposition. The decay of ${ }^{137} \mathrm{Xe}$ and ${ }^{90} \mathrm{Kr}$ with time and increase in the calculated ratio of ${ }^{137} \mathrm{Xe} /{ }^{90} \mathrm{Kr}$ is plotted in Figure $9 .{ }^{137} \mathrm{Cs} /{ }^{90} \mathrm{Sr}$ ratios for the three INGOT samples reflect a maximum nine minute decay of ${ }^{137} \mathrm{Xe}$ and ${ }^{90} \mathrm{Kr}$ (see Table VII). It should be noted that $88 \%$ of ${ }^{90} \mathrm{Kr}$ decays into ${ }^{90} \mathrm{Rb}^{1-}$ and only $12 \%$ into the ${ }^{90} \mathrm{Rb}^{4-}$ isomer (Lederer and Shirley, 1978). The fraction of ${ }^{90} \mathrm{Rb}^{4-}$ generated by independent fission yield will not be transported as gaseous ${ }^{90} \mathrm{Kr}$.

The extreme fractionation of ${ }^{137} \mathrm{Cs}$ and ${ }^{90} \mathrm{Rb}\left({ }^{90} \mathrm{Sr}\right)$ relative to one another and the INGOT radionuclide inventory suggests that late-stage gaseous transport was important in the fractured interval. The volatility of the short-lived precursors must also be emphasized. Radiochemical analyses of solid samples returned from re-entry drilling of the 1981 BASEBALL event documents fractionation of these radionuclides on a field-scale (Thompson, 1995). Calculated partial pressures of $\mathrm{Sb}, \mathrm{Te}$ and $\mathrm{Se}$ are higher relative to rare earth elements, $\mathrm{Zr}$ and $\mathrm{Nb}$ as a function of both oxygen pressure and temperature (Bedford and Jackson, 1965). The final distribution of 


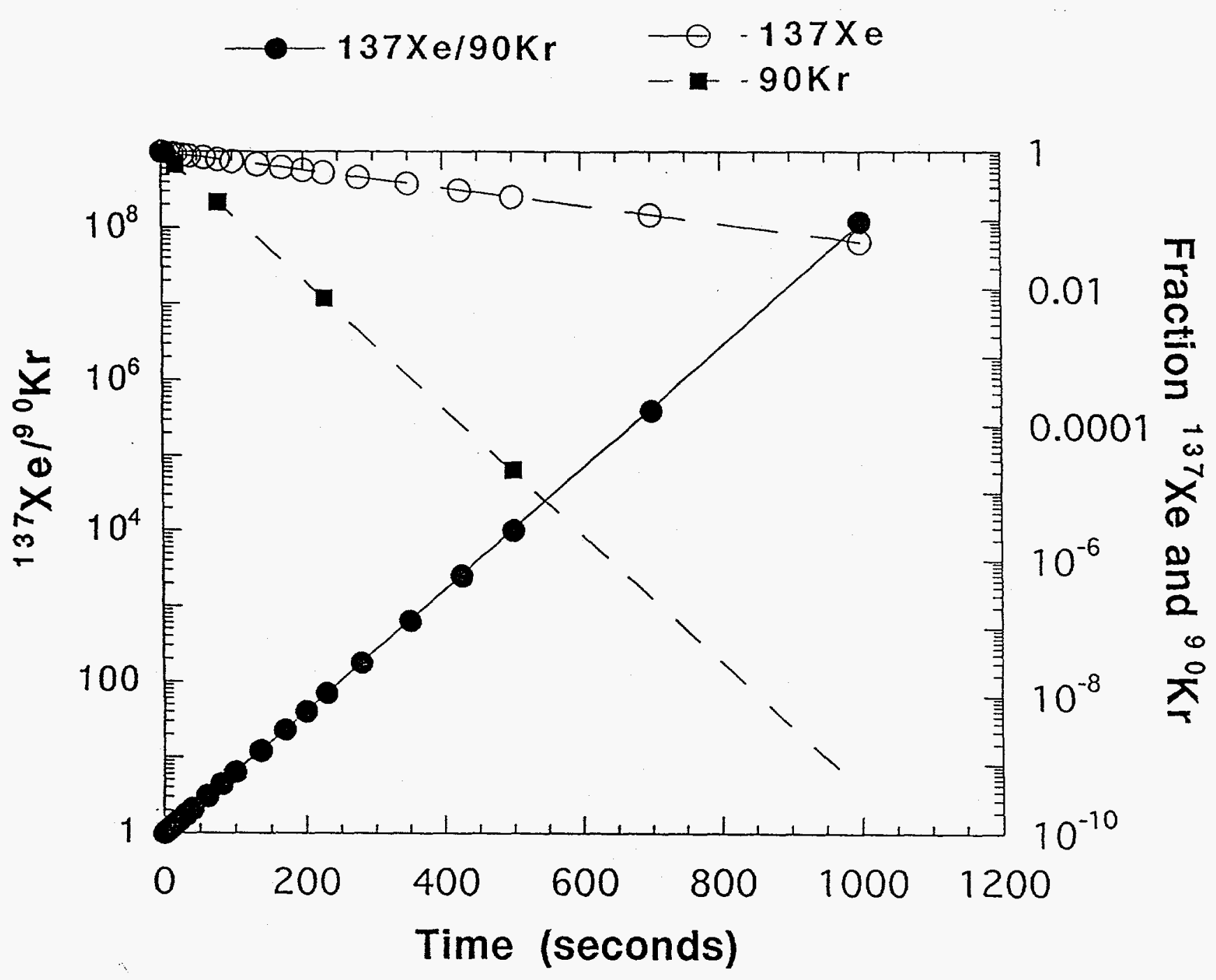

Figure 9: Decay of ${ }^{137} \mathrm{Xe}$ and ${ }^{90} \mathrm{Kr}$ and increase in the calculated ratio of ${ }^{137} \mathrm{Xe} /{ }^{90} \mathrm{Kr}$ with time. The figure assumes equal numbers of initial atoms of ${ }^{137} \mathrm{Xe}$ and $90 \mathrm{Kr}$, ignoring precursors. ${ }^{137} \mathrm{Cs} /{ }^{90} \mathrm{Sr}$ ratio increases a factor of 2 every $\approx 37.6$ seconds. ${ }^{137} \mathrm{Cs} /{ }^{90} \mathrm{Sr}$ ratios measured for the INGOT samples indicate a maximum nine minute decay of ${ }^{137} \mathrm{Xe}$ and ${ }^{90} \mathrm{Kr}$. 
radionuclides may reflect late-stage, low temperature processes following earlier separation of extremely volatile, volatile, intermediate and refractory species from one another. In addition, noncondensible gases (air, $\mathrm{H}_{2}$ and $\mathrm{CO}_{2}$ ) produced or displaced during the explosion may facilitate transport of gaseous species although their exact role has yet to be determined.

The absence of a suite of long-lived refractory fission products argues against the direct injection of an undifferentiated high-temperature plasma at zero time. Earlier investigations at the Nevada Test Site indicated "prompt fracture injection" as a mechanism to transport radionuclides away from the working point. In this conceptual model, a high temperature and high pressure plasma produced by the detonation is injected at zero time along newly created and reactivated fractures surrounding the working point. Nimz and Thompson (1992) attribute radionuclide migration in the unsaturated zone at U-3cn\#5 (BILBY), UE-4g\#2, U-9 ITS U-29, U-3kz (ALEMAN) and UE-2ce to prompt injection. Specifically, Thompson and Gilmore (1991) report on the movement of ${ }^{101} \mathrm{Rh}$ and ${ }^{102} \mathrm{Rh}$ away from the SANDREEF event fired in hole (U-7aq) and its detection in the ALEMAN (U-3kz) emplacement hole 350 meters away. In contrast, at INGOT late-time gaseous transport, rather than prompt injection, is most likely responsible for the migration and distribution of radionuclides outside the cavity.

\section{CONCLUSIONS}

The 1994 INGOT drill-back targeted fractured media adjacent to the edge of the cavity and emphasized the extent of gaseous transport in discrete intervals of enhanced permeability. ${ }^{137} \mathrm{Cs}$ and ${ }^{90} \mathrm{Rb}\left({ }^{90} \mathrm{Sr}\right)$ are ubiquitous fission products produced in underground nuclear explosions and encountered within and adjacent to expended test cavities (Mathews et al., 1994). Studies at INGOT emphasize that, in addition to tritium, these gaseous fission product daughters are abundant and may be encountered ten meters or more away from the cavity edge. Late-time gaseous transport of ${ }^{137} \mathrm{Xe}$ and $90 \mathrm{Kr}$ during an underground nuclear explosion is responsible for the dispersion and subsequent deposition of ${ }^{137} \mathrm{Cs}$ and ${ }^{90} \mathrm{Sr}$. The prevalence of volatile species is significant because these radionuclides may be largely deposited on free surfaces and available to groundwater through processes including ion exchange, desorption and surface-layer alteration associated with dissolution. Dedicated studies at INGOT demonstrate the importance of radiochemical fractionation to partition volatile and gaseous species created during an underground nuclear explosion; further systematic characterization is required before comprehensive statements can be made concerning the distribution and transport of radionuclides near underground test centers. 


\section{ACKNOWLEDGMENTS}

This work could not have been completed without the contributions of many at LLNL, LANL and DOE/NV. For our work at INGOT, the drilling and field expertise of J. Flam, M. Butler, M. Van Dyke, J. Carpenter and J. Thompson is acknowledged. Gamma logs were reduced and interpreted by M. Mathews of LANL and L. Gadeken of Halliburton Energy Services. Copies of the geophysical logs were provided by J. Hearst of LLNL. Our off-NTS groundwater sampling would not have been possible without the cooperation and assistance of D. Wood and R. Laczniak of the USGS, S. Mizell and R. Hershey of DRI, S. Leedom of DOE/NV and Shelby Friend. We also thank private citizens and the City of Alamo, Nevada for providing wellhead access. Logistical support from S. Freiheit of the LLNL Warehouse at the NTS is acknowledged. J. Royval was supported by an Associated Western Universities Undergraduate Fellowship. Report reviews by N. Burkhard of the LLNL Containment Program and A.B. Kersting and B. Esser of the LLNL Isotope Sciences Division are appreciated. S. Lawrence of the Underground Test Area Operable Unit, DOE/NV Environmental Restoration Division (ERD) supported portions of the INGOT field program. Despite budgetary fluctuations, R. Pearl of the HRMP at DOE/NV ERD was always enthusiastic and is thanked for his interest in, and support of, our FY95 program. 


\section{BIBLIOGRAPHY}

Bedford, R.G. and D.D. Jackson, 1965, Volatilities of the Fission Products and Uranium Oxides, Lawrence Livermore National Laboratory, UCRL$12314,37 \mathrm{p}$.

Borg, I.Y., 1974, Radioactivity Trapped in Melt Produced by a Nuclear Explosion, 1975, Nuclear Technology, v. 26, p. 88 - 100.

Buddemeier, R.W. and J.R. Hunt, 1988, Transport of Colloidal Contaminants in Groundwater: Radionuclide Migration at the Nevada Test Site, Applied Geochemistry, v. 3, p 535-548.

Buddemeier, R.W. and D. Isherwood, 1985, Radionuclide Migration Project 1984 Progress Report, Lawrence Livermore National Laboratory, UCRL$53628,71 \mathrm{p}$.

Butkovich, T.R. and A.E. Lewis, 1973, Aids for Estimating Effects of Underground Nuclear Explosions, Lawrence Livermore National Laboratory, UCRL-50929 Rev. 1, 104p.

Butkovich, T.R., 1974, Rock Melt from an Underground Nuclear Explosion, Lawrence Livermore National Laboratory, UCRL-51554, 11p.

Clary, S.L., D.R. McClary, R. Whitney and D.D. Reeves, 1994, Water Resources Data - Nevada, Water Year 1994, United States Geological Survey Water Resources Division, Water-Data Report NV-94-1.

Davisson, M.L., J.M. Kenneally, D.K. Smith, G.B. Hudson, G.J. Nimz and J.H. Rego, January 1994, Preliminary Report on the Isotope Hydrology Investigations at the Nevada Test Site: Hydrologic Resources Management Program, Lawrence Livermore National Laboratory UCRL-ID-116122.

DOE, see U.S. Department of Energy.

Esser, B.K., 1994, The Effect of Bentonite-Based Drilling Fluids on Radiochemical Sampling of Nevada Test Site Groundwaters, Position Paper of the Nuclear Chemistry Division, Lawrence Livermore National Laboratory to the U.S. Department of Energy, Nevada Operations Office, $18 \mathrm{p}$.

Gadeken, L.L., H.D. Smith, Jr., and D.J. Seifert, 1988, Calibration and analysis of borehole and formation sensitivities for gamma ray spectroscopy measurements with multiple radioactive tracers, Log Analyst, v. 29, no. 3, p. 159 - 177. 
Goishi., W., B. Esser, J. Meadows, N. Namboodiri, D. Smith, J. Wild, S. Bowen, P. Baca, L. Olivas, C. Geoffrion, J. Thompson and C. Miller, 1994, Total Radionuclide Inventory Associated with Underground Nuclear Tests Conducted at the Nevada Test Site 1955 - 1992 (U), Los Alamos National Laboratory, LA-CP-94-0222, 242p, SECRET RESTRICTED DATA.

Graham, D.L. and V.G. Johnson, 1991, Effects of Fluid Rotary Drilling on Hydrochemical Sampling Results from Deep Boreholes in Fractured Columbia River Basalt, Journal of Hydrology, v. 128, p. 171-212.

Kenneally, J.M., M.L. Davisson and D.K. Smith, 1995, Multi-Isotopic Evidence for Regional Scale Groundwater Flow into Yucca Flat, Nevada Test Site, Southern Nevada, 1995, Geological Society of America, Abstracts with Programs, v. 27, p. 57.

Knox, J.B., H.A. Tewes, T.V. Crawford, and T.A. Gibson, Jr., 1967, Radioactivity Released from Underground Nuclear Detonations: Source, Transport, Diffusion and Deposition, Lawrence Livermore National Laboratory, UCRL - 50230, 130p, SECRET - RESTRICTED DATA.

Lederer, C.M and V.S. Shirley (eds.), 1978, Table of the Isotopes, 7th ed., John Wiley and Sons, New York, 1523p.

Mathews, M., K. Hahn, J. Thompson, L. Gadeken and W. Madigan, 1994, Subsurface Radionuclide Investigation of a Nuclear Test, Journal of Applied Geophysics, v. 32, p. 279 - 291.

McKinley, P.W., M.P. Long and L.V. Benson, 1991, Chemical Analyses of Water from Selected Wells and Springs in the Yucca Mountain Area, Nevada and Southeastern California, United States Geological Survey Open File Report 90-355.

Nimz, G.J. and J.L. Thompson, 1992, Underground Radionuclide Migration at the Nevada Test Site, U.S. Department of Energy, Nevada Field Office, DOE/NV-246, UC-703, 17p.

Newmark, R.L. and C.S. Schmidt, 1988, U2gg Site Characteristics Report, Lawrence Livermore National Laboratory Containment Program, Report CP 88-89, 41p.

OTA, see U.S. Congress. 
Perfect, D.L., C.C. Faunt, W.C. Steinkampf, and A.K. Turner, 1995, Hydrochemical Data base for the Death Valley Region, California and Nevada, United States Geological Survey Open File Report 94-305.

Smith, D.K., 1995, Characterization of Nuclear Explosive Melt Debris, Radiochimica Acta, v. 69, p. 157 - 167.

Smith, D.K., B.K. Esser and J.L. Thompson, 1995, Uncertainties Associated with the Definition of a Hydrologic Source Term for the Nevada Test Site, Lawrence Livermore National Laboratory, UCRL-ID-120322, 21p.

Thompson, J.L., 1995, Laboratory and Field Studies Related to the Hydrologic Resources Management Program, October 1, 1994 - September 30, 1995, Los Alamos National Laboratory, LA-13064-PR, 27p.

Thompson, J.L. and J.S. Gilmore, 1991, Migration of Fission Products at the Nevada Test Site: Detection with an Isotopic Tracer, Radiochimica Acta, v. 52/53, p. $229-231$.

U.S. Congress, Office of Technology Assessment, 1989, The Containment of Underground Explosions, OTA-ISC-414, Washington, DC, U.S. Government Printing Office, 80p.

U.S. Department of Energy, Nevada Operations Office, Regulatory Acceptance of Well Construction Techniques at the Nevada Test Site, Draft, DOE/NV-XXX, UC-700, Las Vegas, NV.

Waddell, R.K., J.H. Robison and R.K. Blankennagel, 1984, Hydrology of Yucca Mountain and Vicinity, Nevada-California: Investigative Results Through Mid-1983, United States Geological Survey, WaterResources Investigations Report 84-4267.

Wadman, R.E. and W. D. Richards, 1961, Postshot Geologic Studies of Excavations Below Rainier Ground Zero, Lawrence Livermore National Laboratory, UCRL - 6586, 27p.

Winograd, I.J. and W. Thordarson, 1975, Hydrogeologic and Hydrochemical Framework, South-Central Great Basin, Nevada-California, with Special Reference to the Nevada Test Site, United States Geological Survey Professional Paper 712-C, 126p. 